\title{
Probing the clumping structure of giant molecular clouds through the spectrum, polarisation and morphology of X-ray reflection nebulae
}

\author{
Margherita Molaro ${ }^{1}$, Rishi Khatri ${ }^{1,2}$, and Rashid A. Sunyaev ${ }^{1,3}$ \\ 1 Max Planck Institut für Astrophysik, Karl-Schwarzschild-Str. 1, 85741 Garching, Germany \\ e-mail: molaro@mpa-garching.mpg.de \\ 2 Tata Institute of Fundamental Research, Homi Bhabha Road, 400005 Mumbai, India \\ 3 Space Research Institute, Russian Academy of Sciences, Profsoyuznaya 84/32, 117997 Moscow, Russia
}

Received 16 November 2015 / Accepted 14 February 2016

\begin{abstract}
We introduce a new method for probing global properties of clump populations in giant molecular clouds (GMCs) in the case where these act as X-ray reflection nebulae (XRNe), based on the study of the clumping's overall effect on the reflected X-ray signal, in particular on the Fe K- $\alpha$ line's shoulder. We consider the particular case of Sgr B2, one of the brightest and most massive XRN in the Galactic center (GC) region. We parametrise the gas distribution inside the cloud using a simple clumping model with the slope of the clump mass function $(\alpha)$, the minimum clump mass $\left(m_{\min }\right)$, the fraction of the cloud's mass contained in clumps $\left(f_{\mathrm{DGMF}}\right)$, and the mass-size relation of individual clumps as free parameters, and investigate how these affect the reflected X-ray spectrum. In the case of very dense clumps, similar to those presently observed in Sgr B2, these occupy a small volume of the cloud and present a small projected area to the incoming X-ray radiation. We find that these contribute negligibly to the scattered X-rays. Clump populations with volume-filling factors of $>10^{-3}$ do leave observational signatures, that are sensitive to the clump model parameters, in the reflected spectrum and polarisation. Future high angular resolution X-ray observations could therefore complement the traditional optical and radio observations of these GMCs, and prove to be a powerful probe in the study of their internal structure. Clumps in GMCs should further be visible both as bright spots and regions of heavy absorption in high resolution X-ray observations. We therefore also study the time-evolution of the X-ray morphology, under illumination by a transient source, as a probe of the 3D distribution and column density of individual clumps by future X-ray observatories.
\end{abstract}

Key words. Galaxy: center - scattering - polarization - ISM: structure

\section{Introduction}

Understanding the internal structure of giant molecular clouds (GMCs), which is driven by the interplay of turbulence, selfgravitation, and magnetic fields, is crucial when studying star formation processes in galaxies. It is, in fact, inside GMCs that dense, gravitationally unstable regions of gas, known as prestellar cores, form and collapse to give birth to stars (Williams et al. 2000).

Direct and exhaustive studies of the internal structure of GMCs are severely limited by issues of spatial and mass resolution when observing small-scale gas substructures. This is particularly true for GMCs that are located at a great distance, for example those found in the central molecular zone (CMZ), the region within the central $\sim 400 \mathrm{pc}$ by $\sim 80 \mathrm{pc}$ of the Galaxy (Langer et al. 2015). Dense regions inside GMCs, often studied as discrete objects loosely classified as clumps and cores, span spatial ranges of $0.2-2 \mathrm{pc}$ and $0.02-0.4 \mathrm{pc}$ and mass ranges of $10-10^{3} M_{\odot}$ and $0.3-10^{2} M_{\odot}$, respectively (Draine 2011). At distances comparable to that from the Sun to the Galactic centre (GC), subarcsec angular resolution is therefore required to study these structures in detail. Despite the challenge that such highresolution observations pose, obtaining a clear and complete picture of the overall properties of the clump and core populations in GMCs remains a significant effort in developing theoretical models of star formation (Williams et al. 2000).
In this paper, we suggest a new method for probing global properties of the clump and core population in GMCs in the case where these act as X-ray reflection nebulae (XRNe), based on the study of their overall effect on the reflected X-ray signal.

The X-ray emission from XRNe is composed both of a continuum, shaped by the interplay of scattering and absorption of the illuminating X-rays by atoms and molecules in the GMC, and by characteristic spectral features in the $\mathrm{keV}$ regime. The latter are caused by the emission of fluorescent photons by heavy elements, which follows the photoionisation of tightly bound electrons by hard X-rays. The inelastic scattering of fluorescent photons down to lower energies results in a characteristic increase in the continuum at energies lower than the fluorescent features - the so called "shoulder". This feature is most easily visible in the case of bright fluorescent lines, such as the Fe K- $\alpha$ line. Therefore, in the case of a small optical depth $(\tau<1)$, the intensity of the reflected continuum, fluorescent lines and shoulders will depend on $\sim \tau_{T}, \tau_{\text {abs }}$ and $\tau_{\mathrm{T}} \times \tau_{\text {abs }}$ respectively (where $\tau_{\mathrm{T}}$ and $\tau_{\mathrm{abs}}$ are the Thomson scattering and photoabsorption optical depths).

Fluorescent emission following illumination by $\mathrm{X}$-rays was predicted by Sunyaev et al. (1993) in support of the claim that GMCs that surround the Galactic centre (GC) should act as XRNe of past flares of Sgr A*, the super-massive black hole located at the centre of the Galaxy. If this were the case, then part of the diffuse, hard, X-ray emission observed from these GMCs 
should be composed of a flux in the neutral Fe fluorescent line energy $(6.4 \mathrm{keV})$, which is caused by the imprint of past, prequiescent activity of $\mathrm{Sgr} \mathrm{A*}$ on the present-day (due to time delays) X-ray emission of GMCs that are located in its proximity. A high, time-varying flux at $6.4 \mathrm{keV}$ was indeed observed in the GMC Sgr B2 by Koyama et al 1996, and has been extensively studied ever since (Murakami et al. 2000; Muno et al. 2007; Inui et al. 2009; Ponti et al. 2010, 2013; Terrier et al. 2010; Capelli et al. 2012; Nobukawa et al. 2011; Gando Ryu et al. 2013; Clavel et al. 2013; Zhang et al. 2015). Scattered flux from Sgr B2 in hard X-rays has also been detected using Integral (Revnivtsev et al. 2004). Similarly, other GMCs in the CMZ (Murakami et al. 2001; Marin et al. 2015) have since been shown to act as XRNe. An alternative scenario to the reflection of past $\mathrm{Sgr} \mathrm{A}^{*}$ flares, which explains the $6.4 \mathrm{keV}$ emission in Sgr B2 as the result of the interaction of low-energy cosmic ray electrons with matter in the GMCs, has also been proposed (Valinia et al. 2000; Yusef-Zadeh et al. 2002; Yusef-Zadeh 2013; Dogiel et al. 2009). Both scenarios will equally be affected by the clumping of gas inside Sgr B2 discussed in this work. The two cases can however be differentiated by considering of the different energy deposition rates resulting from either X-ray or cosmic ray propagation, and by the speed of propagation of the Fe K- $\alpha$ front. In particular, the observation of a superluminal echo (Sunyaev \& Churazov 1998; Sunyaev et al. 1999; Ponti et al. 2010) in the $6.4 \mathrm{keV}$ brightness cannot be explained by any model of line excitation other than the one resulting from illumination by an external source. In addition, studies of the polarisation and spectral absorption edges in the X-ray continuum, both of which would be expected in the reflection scenario but not in the cosmic-ray one, can further help distinguish the two effects. NuSTAR-type hard X-ray grazing incidence telescopes should be able to detect absorption edges for a few heavy elements, including $\mathrm{Fe}$ and $\mathrm{Ni}$.

Both the continuum and fluorescent spectral features of the reflected X-ray spectrum are dependent on the structure and composition of the gas itself, as well as on the properties of the $\mathrm{X}$-ray source illuminating the gas, and on the relative position of the source and the gas with respect to the observer. They therefore contain a wealth of information both on the source itself and on the gas structures surrounding it.

Several models (Sunyaev \& Churazov 1998; Murakami et al. 2000; Churazov et al. 2002; Odaka et al. 2011; Marin et al. 2014a,b, 2015; Marin 2015) have been developed to simulate the reflection of Sgr A* flares by Sgr B2, the brightest of the CMZ $\mathrm{XRNe}$. These models considered different relative positions of the GMC with respect to the source, different total masses of the GMC and different density gradients of its gas.

In this work, we wish to expand on these models to investigate how more realistic models of the substructure of molecular clouds, in particular their clumpiness, would affect the reflected $\mathrm{X}$-ray signal. (From now on we refer to both clumps and cores as clumps for simplicity, as the latter term merely refers to the low-mass end of the same population of overdensities.)

We use a 3D grid-based Monte Carlo radiative transfer code to compute the reflected energy spectrum and polarisation of Sgr B2's X-ray emission. In Sect. 2 we discuss the physical processes accounted for and the Monte Carlo method used in our calculations. In Sect. 3, we discuss the models for Sgr B2 considered, including the parameters used and the assumptions made in simulating its clump population (Sect. 3.1). In Sect. 4 we discuss the results obtained.

Finally, in Sect. 5, we discuss the time-variability of the $\mathrm{X}$-ray morphology of XRNe in the case of clumps. We suggest that, under illumination by a non-persistent, flaring source such as $\mathrm{Sgr} \mathrm{A}^{*}$, the evolution of reflected X-ray intensity can reveal information about the location of the clumps along their line of sight, and therefore about the 3D distribution of these substructures inside GMCs.

Even though we simulate the particular case of Sgr B2, our results are more generally applicable to other GMCs in the Galaxy when illuminated by other X-ray sources such as X-ray binaries (XRBs). Notice that in such cases, however, the polarisation of the primary $\mathrm{XRB}$ radiation due to scattering onto the corona and accretion disk, as well as due to relativistic effects, would need to be carefully taken into account (Meszaros et al. 1988; Haardt \& Matt 1993; Poutanen \& Vilhu 1993; Taverna et al. 2014). Assumptions on the polarisation state of Sgr A* flares assumed in our calculations are discussed in Sect. 4.

\section{Monte Carlo simulation of X-ray propagation in inhomogeneous media}

In this section, we describe the physical processes accounted for to simulate the propagation of X-ray photons in neutral gas (Sect. 2.1) and the Monte Carlo radiative transfer code used (Sect. 2.2).

\subsection{X-ray interaction with neutral matter}

$\mathrm{X}$-rays interaction with atoms and molecules in the interstellar gas takes place through two processes: scattering and absorption through photoionisation.

Scattering processes of X-ray photons on bound electrons are classified as Rayleigh in the case of elastic scattering, Raman for scattering that results in the excitation of electrons in the atom or molecule, and Compton for scattering that results in the ionisation of the atom (Sunyaev \& Churazov 1996). In our code, we account for these scattering processes on neutral HI, H2 and He using the results for the doubly differentiated cross-section of Vainshtein et al. (1998). The contribution of heavier elements to the total scattering cross-section, for which such results are not currently available, is accounted for by approximating the interaction of X-rays with their electrons as if they were unbound. We also include the effect of polarisation in Rayleigh and Compton scattering processes using the prescription of Namito et al. (1993). For simplicity, we ignore the polarisation dependence of Raman scattering, which can cause a depolarisation of the scattered radiation (Allemand 1970; Szymanski 1970). Raman scattering contributes less than $1 \%$ to photons observed after a single scattering for the geometry considered in this work (average angle $\sim 90^{\circ}$ ), and will only contribute $10-15 \%$ in a narrow $\left(\sim 20^{\circ}\right)$ angular range in the scattering of secondary photons. This approximation therefore will not significantly affect our results, although it may affect the polarisation of the reflected spectrum under a different geometry.

Photoionisation, on the other hand, takes place through the ionisation by X-ray photons of tightly bound electrons in the atoms' innermost shells, which results in the release of a free electron. We use cross-sections from Verner \& Yakovlev (1995), and include both K- and L-shell photoabsorption. The unstable electron configuration of the ionised atom prompts the filling of the K-shell vacancy by an electron in one of the higher energy levels, which causes a release of energy. This energy can either be released through the emission of a photon in the X-ray range (fluorescence) or be transferred to another electron, which is then ejected from the atom (Auger effect). The probability of either 
process taking place varies depending on the atomic configuration and on the original energy level of the electron that fills the $\mathrm{K}$-shell vacancy. The probability of fluorescence is called the radiative yield $(Y)$. In our calculation, $\mathrm{K}$-shell fluorescence yields are taken from Krause et al 1979 , and $\mathrm{K}_{\beta}$-to- $\mathrm{K}_{\alpha}$ ratios are taken from Ertugral et al 2007. $\mathrm{K}_{\alpha 2}$-to- $\mathrm{K}_{\alpha 1}$ ratios are decided by the degeneracy of $2_{p_{1 / 2}}$ and $2_{p_{3 / 2}}$ levels, which we fix to 0.5 . The energies of the fluorescent lines are taken from Thompson et al. (2001).

We assume a chemical composition of the Sgr B2 cloud given by a factor of 1.5 compared to protosolar abundance, as estimated by Lodders (2003).

\subsection{Photon weighing method}

We use a Monte Carlo grid-based radiative transfer code to simulate the propagation of X-ray photons through a cloud of complex internal structure, containing both diffuse and dense regions. We use an octree-based approach (Meagher 1980) to grid the cloud's internal structure, which allows us to have finer grids in high density regions.

The Monte Carlo code makes use of the Pozdnyakov et al. (1983) prescription for Monte Carlo methods of X-ray propagation. This applies a weight-based approach to the radiative transfer, in which photon packages, rather than individual photons, are followed. Each package is described by a statistical weight $w$, which reflects the relative probability of photons undergoing different types of interactions, a position, $\boldsymbol{r}$, a direction of travel, $\boldsymbol{\Omega}$, and an energy, $h v$.

Photon-packages are initially emitted by the source with weight 1 . Their starting position is the source's own position, and their energy is randomly sampled from the source's spectrum. Finally, their initial direction is randomly sampled from an isotropic distribution (assuming the source radiates isotropically).

To compute the propagation of photon packages in our grid, we estimate the relative probability of different processes occurring at each step. A photon package $\boldsymbol{P}$, found in a given gridcell $g$, has, in fact, a probability of:

- escaping the grid-cell $g,(L)$;

- not escaping the grid-cell $g,(1-L)$;

and, if not escaping the grid-cell $g$, a probability of:

- being scattered, $\left(p_{\text {scatt }, Z}\right)$;

- being absorbed by a $\mathrm{K}$ or an $\mathrm{L}$ shell, $\left(p_{\mathrm{abs}, Z}=p_{\mathrm{Kion}, Z}+\right.$ $\left.p_{\text {Lion, }}\right)$.

By considering photon packages rather than single photons, we are able to account for all the above events at once by splitting the initial photon package weight $w$ as follows:

- $w_{u}=w L$ is the probability of $\boldsymbol{P}$ crossing the grid-cell without undergoing any interaction;

- $w_{s}=w(1-L) p_{\text {scatt }, Z}$ is the probability of $\boldsymbol{P}$ undergoing a scattering event inside the grid-cell;

- $w_{\text {fluo }, Z}=w(1-L) p_{\text {Kion, } Z} Y$ is the probability of $\boldsymbol{P}$ photoionising the $\mathrm{K}$ shell of element $Z$, resulting in a fluorescent emission.

We can account for these events by assigning their weight to secondary packages $\boldsymbol{P}_{\mathrm{u}}, \boldsymbol{P}_{\mathrm{s}}$, and $\boldsymbol{P}_{\mathrm{f}, Z} \mathrm{~s}$, which will represent the relative probability of each one of those physical events taking place. Parameters $\boldsymbol{r}, \boldsymbol{\Omega}$ and $h v$ in $\boldsymbol{P}_{\mathrm{u}}, \boldsymbol{P}_{\mathrm{s}}$, and $\boldsymbol{P}_{\mathrm{f}, Z} \mathrm{~s}$ of course have to be updated, each according to the physical processes that are relevant for that secondary package.

Once all the parameters have been updated, the calculation is iterated by taking each one of the secondary packages as an initial package $\boldsymbol{P}$, and so on, for the secondary packages that are then produced. To limit the number of secondary photon packages that the code has to follow, we define a statistical threshold $\epsilon$, below which secondary packages are discarded.

We note that other possible events, for example the emission of an Auger electron, which is not listed above, as well as any secondary processes they may cause, will not result in the emission of X-ray radiation, and can therefore be safely ignored in the processing of the X-ray radiation field. They will however contribute to the deposition of X-ray energy to the interstellar gas. The convergence of the code with respect to the threshold $\epsilon$ and other parameters was verified, as well as the consistency of our results with those of Odaka et al. (2011; for the energy spectrum) and Churazov et al. (2002; for the polarisation spectrum).

\section{Sgr B2 model}

Sgr B2 is not only one of the most massive, but also one of the most complex molecular structures observed in our Galaxy, containing $\sim 10 \%$ of the molecular mass in the CMZ (Gordon et al. 1993). It is also one of the brightest XRNe observed, making it an ideal target for our study.

Located at a projected distance from the GC of $\sim 100 \mathrm{pc}$, its exact position on the line of sight still remains uncertain. In a coordinate system in which the GC is located at $(0,0,0)$, the observer at the Sun's location $(0,-8 \mathrm{kpc}, 0)$, and the Galactic longitude is defined in the direction of the positive $y$ axis, we assume a fixed position of the GMC at $(0,100 \mathrm{pc}, 0)$, so that the angle between the source, the cloud and the observer is $\sim 90^{\circ}$. Discussions on how different geometries affect the reflected X-ray signal can be found in Churazov et al. (2002), Odaka et al. (2011) and Marin et al. (2014a).

Studies of the large scale morphology of Sgr B2 (Lis \& Goldsmith 1990) show it is surrounded by a diffuse gas envelope, extending up to $22.5 \mathrm{pc}$ in radius, with a near-uniform density of $n_{\mathrm{H} 2} \sim 10^{3} \mathrm{~cm}^{-3}$. What is generally referred to as the Sgr B2 cloud, and where most of the reflected $\mathrm{X}$-ray signal originates, is a dense region within this envelope, of density $n_{\mathrm{H} 2} \sim 10^{4-5} \mathrm{~cm}^{-3}$ and extending out to a radius of 10 pc (Hasegawa et al. 1994). Within this region, multiple dense clumps can be observed. The Sgr B2 GMC contains three well-known dense clumps, named B2(N), Sgr B2(M) and Sgr B2(S). These are known to host clusters of compact HII regions, which provide evidence for starformation activity within this GMC (Gordon et al. 1993). Two of these cores, SgrB2(N) and SgrB2(M) (with masses of $3313 M_{\odot}$ and $3532 M_{\odot}$ (Qin et al. 2011) and radii $0.47 \mathrm{pc}$ and $0.62 \mathrm{pc}$ (Etxaluze et al. 2013) respectively), have been resolved at a subarcmin scale in X-rays (Zhang et al. 2015) and at a subarcsec scale using the Submillimeter Array (SMA) by Qin et al. (2011). The high angular resolution reached by the latter study reveals a remarkable difference in the internal structure of the two cores, with SgrB2(M) appearing to be highly fragmented into 12 sub-cores and SgrB2(N) appearing to be divided into only two sub-cores, one of which contains most of the mass. The very different morphologies of the two have been speculated as evidence in support of the idea that the two cores may represent different evolutionary stages of basically the same core, given that $\operatorname{SgrB} 2(\mathrm{~N})$ and $\operatorname{SgrB} 2(\mathrm{M})$ are of comparable size and mass. 
In our calculations, we assume Sgr B2 to have a mass of $M_{\mathrm{B} 2}=2.5 \times 10^{6} M_{\odot}$ within radius $10 \mathrm{pc}$, and a diffuse $\mathrm{H} 2$ envelope around it as described above. In our calculations, we approximate this envelope following Odaka et al. (2011)'s prescription, by considering an initial absorption to the incoming spectrum owing to a column density of $N_{\mathrm{H} 2}=6 \times 10^{22} \mathrm{~cm}^{-2}$. We note that this initial absorption will only affect the incoming spectrum below $\sim 4 \mathrm{keV}$ energies.

Given this mass and size, we then consider different possible models for the clump population inside the cloud, as discussed in the next section. The gas is assumed to be cold and neutral.

In our model, the optical depth of the gas located between Sgr A* and Sgr B2 is assumed to be negligible. This is a clear simplification of the gas distribution in the Galactic centre region, and there is indeed a possibility that part of other important molecular structures located in the vicinity of Sgr B2, such as the Bridge, the MC 2, or the G0.11-0.11 cloud, could intervene between the two (Capelli et al. 2012; Clavel et al. 2013; Ponti et al. 2010, 2014). If the optical depth of the intervening gas were significant, it would obviously contribute towards the absorption and scattering of the incoming radiation. This would modify the radiation reaching Sgr B2, whose illumination would then become dependent on the morphology of the intervening gas. In particular, different parts of Sgr B2 would, in this case, be illuminated by X-rays described by different spectral energy distributions. We however do not consider such complications in the present work.

\subsection{Simulating Sgr B2's clump population}

Since the outset of this field, statistical descriptions of the internal structure of GMCs have been formulated in terms of discrete over-dense regions within GMCs (i.e. clumps and cores). More recently, a growing number of studies have adopted a different approach and described these density fields in terms of fractals, placing emphasis on the self-similarity of structural features at all scales (see Williams 1999). In our work we will use the former approach.

The statistical study of these discrete over-dense regions has been applied to a number of nearby Galactic plane GMCs, such as Orion and W51 (e.g. Parsons et al. 2012). This has led to the formulation of standard population properties and relations, which have been generalised from studies of Galactic molecular clouds (e.g. Larson 1981): the clump mass function $(\mathrm{CMF})$ and mass-size relation of individual clumps. These studies, along with simulations, suggest that similarities exist both in the CMF and mass-size relations between different GMCs, and therefore point towards a universal structure of GMCs in the Galactic plane (see Sects. 3.1.1 and 3.1.2). The internal structure of GMCs in the CMZ, on the other hand, is likely to differ greatly from that observed in Galactic plane GMCs, because of the extreme environment, both in density (Lis \& Goldsmith 1990) and pressure (Kruijssen et al. 2014). Understanding how the clump population of these objects differs from the ones found in Galactic plane GMCs, would, therefore, provide an important insight into how environmental factors affect the process of internal structure formation.

As previously discussed, the studies of GMCs in the CMZ are rather more difficult to perform, on account of the large distance and mass of these complexes: catalogues of clump and core populations in these GMCs are known to be incomplete, and therefore don't allow for a reliable estimate of their statistical properties.
In our calculations, we consider the different possible shapes of the CMF and mass-size relation (see Sects. 3.1.1 and 3.1.2 respectively). We also consider the different levels of fragmentation of the cloud into clumps by considering different possible values of the dense gas-mass fraction, or the fraction of the cloud's mass found in clumps, $f_{\mathrm{DGMF}}$ (see Fig. 1). In particular, we investigate the effects of the following clump population model parameters on the XRNe's X-ray emission:

- slope of the clump mass function $(\alpha)$;

- minimum clump mass in the clump mass function $\left(m_{\min }\right)$;

- fraction of the total cloud's mass found in clumps, or dense gas mass fraction $\left(f_{\mathrm{DGMF}}\right)$;

- the mass-size relation of individual clumps $(m=$ $\left.m_{\text {norm }}(r / p c)^{\gamma}\right)$.

The simulation of different clump population models for Sgr B2 is then performed as follows: given a $f_{\mathrm{DGMF}}$ and $\mathrm{CMF}$, we sample the clumps' masses from the CMF in the mass range $m_{\text {min }}-m_{\max }$, with $m_{\max }=10^{4} M_{\odot}$, until we obtain a total mass of $M_{\mathrm{DGMF}} \sim f_{\mathrm{DGMF}} \times M_{\mathrm{B} 2}$. We then proceed to assign each clump with a size that is based on its mass assuming a mass-size relation. Finally, we uniformly distribute the resulting clumps inside the cloud and calculate the interclump density using the "leftover" mass, $M_{\text {interclump }} \sim\left(1-f_{\mathrm{DGMF}}\right) \times M_{\mathrm{B} 2}$ and the "empty" (i.e. not occupied by clumps) volume, assuming the interclump density is homogeneously distributed.

In the following sections, we discuss the details of this procedure, together with the range of parameters that we considered and the assumptions made in doing so.

\subsubsection{Clump mass function}

The clump mass function (CMF) of clump populations, which can be observed in Galactic plane GMCs, takes the following form (McKee \& Ostriker 2007):

$$
m \frac{\mathrm{d} N}{\mathrm{~d} m} \propto m^{-\alpha} .
$$

The slope of the clump mass function is similar to that for GMCs as a whole, possibly because both are determined by turbulent processes within larger, gravitationally bound systems (McKee \& Ostriker 2007). The parameter $\alpha$ takes different values in different mass ranges. The low mass end of the function is known as the core mass function. Its shape is particularly important in relation to the initial mass function (IMF) of stellar populations (McKee \& Ostriker 2007). Evidence of similarity in the core mass function with the IMF were first studied by Nutter \& WardThompson (2007) in the Orion complex, which for the first time observed a turnover at $\sim 1.3 M_{\odot}$, which mimics the turnover seen in the stellar IMF at $0.1 M_{\odot}$. Fitting the data with a three-part power law function similar to that observed in the stellar IMF, they obtain the following parameters:

$$
\alpha= \begin{cases}-0.3 & \text { if } 0.4 M_{\odot}<m<1.3 M_{\odot} \\ 0.3 & \text { if } 1.3 M_{\odot} \leq m \leq 2.4 M_{\odot} \\ 1.35 & \text { if } m>2.4 M_{\odot}\end{cases}
$$

for clump mass ranges below $100 M_{\odot}$. The physical significance of the turnover mass is not clear, since the same work highlights that similar studies in the lower-mass and nearer cloud complex $\rho$ Ophiuchi showed no turnover in their CMF (Motte et al. 1998). Later studies on the low-mass end of the CMF in the 
M. Molaro et al.: Internal structure of XRNe
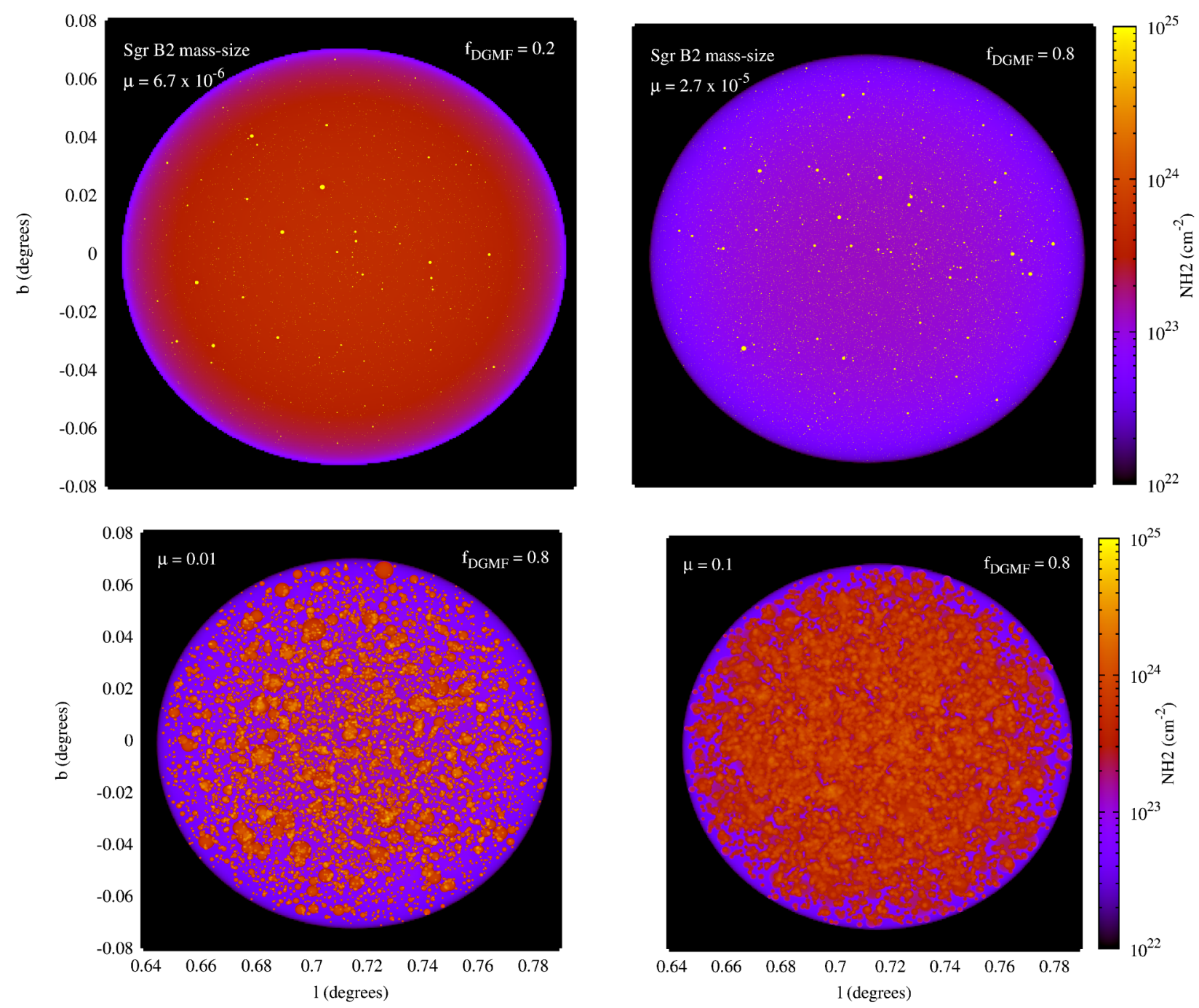

Fig. 1. Cumulative column density $\left(N_{\mathrm{H} 2}\right)$ along line of sights, excluding ISM and diffuse envelope contributions. The maps show Sgr B2 models with fixed parameters $\alpha=1.35, m_{\min }=10 M_{\odot}$ and Sgr B2 mass-size relation, and two values of $f_{\mathrm{DGMF}}$. The concentration of mass into small dense regions reduces the interclump density, as seen from the figures. In the case of $f_{\mathrm{DGMF}}=0.8$, we also show clump populations with mass-size relations tuned to obtain a volume-filling fraction of $\mu=0.01$ and $\mu=0.1$. At a distance to the centre of Sgr B2 of $\sim 8000 \mathrm{pc}, 1$ arcmin corresponds to a size of $\sim 2.3 \mathrm{pc}$.

Aquila rift cloud complex also confirmed a variation in this parameter: Könyves et al. (2010) found a turnover mass of $\sim 0.6 M_{\odot}$ in the starless core sample, and $\sim 0.9 M_{\odot}$ prestellar (starless and gravitationally bound) sub-sample.

The similarity of the CMF with the Salpeter (1955) stellar IMF has been further confirmed in higher mass ranges by Tsuboi \& Miyazaki (2012; range $>900 M_{\odot}$ ) and Parsons et al. (2012; range $\left.>200 M_{\odot}\right)$.

Despite the fact that the CMF appears to be consistent throughout Galactic plane GMCs, the extreme environment of Sgr B2, as previously discussed, suggests the CMF for this GMC could be significantly different. Unfortunately, because of a lack of exhaustive data, how this function could differ is not obvious. We therefore adopt the Nutter \& Ward-Thompson (2007) three-part power-law fitting, but consider different $\alpha$ values in the highest mass interval. In particular, we consider $\alpha=1.35$ (i.e. Galactic plane value), 1, 0.5,1.8 (see Fig. 2). We sample this range up to clump masses $10^{4} M_{\odot}$, to be consistent with the observed massive cores in Sgr B2 (e.g. Qin et al. 2011). We maintain the low-mass threshold of the CMF as a parameter in the model, $m_{\min }$, and investigate its effect on the reflected $\mathrm{X}$-ray signal.

\subsubsection{Clumps mass-size relation}

The mass-size relation of individual clumps is particularly important for our study, as it determines the volume-filling fraction, $\mu$, of the clump population given its $\alpha, m_{\min }$ and $f_{\mathrm{DGMF}}$ parameters. The volume-filling fraction, in return determines how effectively clumps "hide" part of the cloud's mass, by concentrating it into small volumes which X-rays have a low probability of intercepting.

For clumps formed inside GMCs via turbulent fragmentation, we would expect to find a mass-density, or similarly a masssize, relation (Donkov et al. 2011). A first suggestion of this relation based on observations was formulated in the seminal paper of Larson (1981), which claimed a universal power-law masssize relationship should describe all clouds and clumps found in the Galaxy. This is commonly referred to as "Larson's third law", and it suggested that the relation should go as

$m=m_{\text {norm }} r^{\gamma}$

with $\gamma=2$. Since being first formulated, this empirical observation has been extensively studied both in observations (e.g. Kauffmann et al. 2010; Lombardi et al. 2010) and in simulations 


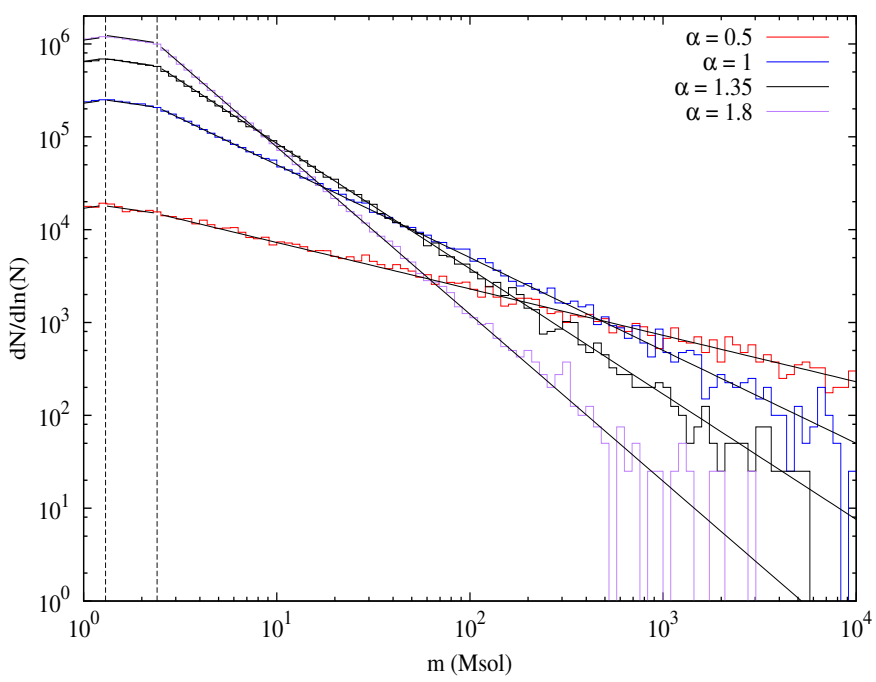

Fig. 2. Clump mass functions for different $\alpha$ with $f_{\mathrm{DGMF}}=0.4$. The grey solid lines show the distributions sampled, while the coloured lines show the actual realisation. The vertical lines show the values at which the $\mathrm{CMF}$ changes in slope. As the slope of the function steepens, more clumps are selected from the lower-mass range. This leads to a progressive increase in the numerical noise in the sampled population at higher masses with increasing $\alpha$, as seen in the plot.

(Shetty et al. 2010; Donkov et al. 2011), which all find a deviation from the power of 2 originally found by Larson (1981). The latter two studies find that on scales $<1 \mathrm{pc}$, the masssize relation studied in 11 different GMC structures in the Galactic plane, can be described as $m(r)=400 M_{\odot}(r / p c)^{1.7}$ and $m(r)=380 M_{\odot}(r / \mathrm{pc})^{1.6}$ respectively. By looking at these clouds individually, Lombardi et al. (2010) find that these clouds have quite similar exponents, but rather different normalisation masses (ranging from 170 to $710 M_{\odot}$ ).

Although these studies haven't been performed for the clump population of Sgr B2, we can use observational data that is currently available to get a rough estimate of what this relation would look like for this GMC.

We use the data available from the 14 cores within Sgr B2(N) and SgrB2(M) which are resolved in the subarsec observations of Qin et al. (2011; see Sect. 3) to infer a mass-size relation for Sgr B2. Assuming a homogeneous density distribution inside the clumps, we fit the power law to these observations and find:

$m(r)=4.68 \times 10^{5} M_{\odot}(r / \mathrm{pc})^{1.77}$

which we refer to henceforth as the "Sgr B2 mass-size relation". We find that the exponent is consistent with the results of Lombardi et al. (2010), but that the normalisation mass is considerably higher. This is somehow to be expected, considering that the average density in Sgr B2 is considerably higher than that found in Galactic plane GMCs. We note that the range of sizes of these cores is considerably below the Lombardi et al. (2010) range. Furthermore, the assumption of gas being homogeneously distributed inside the clumps is also a clear simplification, which could have an impact on our results (see Odaka et al. 2011, for considerations on the effect of density gradient on reflected X-ray emissions). In our model, we assume this relation, inferred in a somewhat limited mass range, holds for all possible clump masses.

As already mentioned, the mass-size relation is particularly important for our study as it effectively determines the volumefilling factor of a given population. The volume-filling factor, $\mu$,

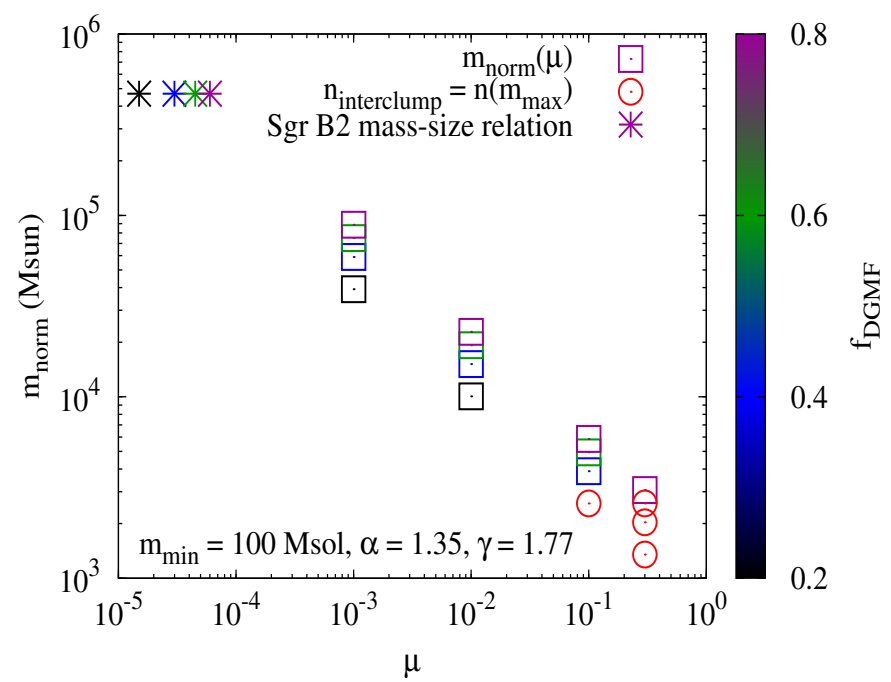

Fig. 3. Normalisation parameter $m_{\text {norm }}$ in the mass-size relation as a function of the volume-filling factor $\mu$, calculated using Eq. (5), for different $f_{\mathrm{DGMF}}$ at fixed $\alpha=1.35, m_{\min }=100 M_{\odot}$ and $\gamma=1.77$. We also show the $\mu$ values, obtained using the fixed Sgr B2 relation. Note that for high $\mu$ and low $f_{\mathrm{DGMF}}$ values, we obtain mass-size relations yielding clump populations where the least dense clump, given by the clump with the highest mass $\left(m_{\max }\right)$, is less dense than the interclump density $n_{\text {interclump }}$, in contradiction with the very definition of a clump as an overdensity; we clearly indicated these models in red in the plot. We will use the $f_{\mathrm{DGMF}}$ value that allows us to explore a widest possible range of $\mu, f_{\mathrm{DGMF}}=0.8$, to investigate the impact of increasing $\mu$ on the reflected X-ray signal (see Fig. 7).

can be expressed in terms of other clump population parameters as follows:

$\mu=\frac{4 \pi}{3 V_{\mathrm{SgrB} 2}} \frac{C}{m_{\mathrm{norm}}^{3 / \gamma}} \frac{\left[m^{-\alpha+3 / \gamma}\right]_{m_{\min }}^{m_{\max }}}{-\alpha+3 / \gamma}$

where

$C= \begin{cases}M_{\mathrm{DGMF}}(1-\alpha)\left(\left[m^{-\alpha+1}\right]_{m_{\min }}^{m_{\max }}\right)^{-1} & \text { if } \alpha \neq 1 \\ M_{\mathrm{DGMF}}[\ln m]_{m_{\min }}^{m_{\max }} & \text { if } \alpha=1 .\end{cases}$

Using Eq. (5), and assuming the mass-size relation given in Eq. (4), we obtain a maximum filling fraction of only $10^{-4}$ (see Fig. 3) among all $f_{\mathrm{DGMF}}, \alpha$ and $m_{\min }$ values considered. Therefore the probability of X-rays intercepting the clumps is extremely low. Hence, we don't expect parameters related to the mass distribution of the clumps, i.e. $\alpha$ and $m_{\min }$, to significantly affect the $\mathrm{X}$-ray signal when assuming this mass-size relation. We also consider how the X-ray signal could be affected by increasingly large values of $\mu$, by varying the $m_{\text {norm }}$ value in the mass-size relation accordingly (see Sect. 4.2).

The mass-size relation can be alternatively expressed as a column density-mass relation to the centre of the cloud. Defining $\tau_{\mathrm{H} 2}=r n_{\mathrm{H} 2} \sigma$, this takes the following form:

$\tau_{\mathrm{H} 2}=m_{\text {norm }}^{2 / \gamma} \frac{3 \sigma}{m 4 \pi} m^{-2 / \gamma+1}$.

The effect of the mass-size relation on the clumps' contribution to the column density of the GMC is shown in Fig. 1.

\subsubsection{Dense gas mass fraction}

Studies of this parameter in Galactic GMCs, e.g. in W51 (Battisti \& Heyer 2014; Ginsburg et al. 2015), show a variety of possible 
stages of fragmentation in different clouds, which reflects the different evolutionary stages GMCs can be found in. We therefore span a wide range of $f_{\mathrm{DGMF}}$ values: $f_{\mathrm{DGMF}}=0.2,0.4,0.6$, and 0.8 .

\subsubsection{Spatial distribution of clumps}

In our model we assume clumps are not overlapping and are isotropically distributed inside the cloud.

The latter assumption is a clear simplification of what is currently being observed in CMZ GMCs (e.g. Chen \& Ostriker 2015). In the case of very low volume-filling factors, as in the case of clumps that follow the Sgr B2 mass-size relation, this assumption should have a negligible effect on the reflected signal. On the other hand, this may become relevant in the case of larger volume-filling factors. The percolation of the X-ray photons is dependent on the projected area filling on the plane perpendicular to the source-cloud direction, rather than on the volumefilling factor itself. The maximum possible projected area occupied by the clumps, in the case where no line of sight from the source intercepts more than one clump, is $\propto \pi \sum_{i=1}^{N} v_{i}^{2 / 3}$, where $v_{i}$ is the volume of the single clump $i$, and $N$ is the total number of clumps. The minimum possible project area on the other hand, in the case where all clumps are centred along a single line of sight, is $\propto \pi v\left(r_{\max }\right)^{2 / 3}$, where $v\left(r_{\max }\right)$ is the volume of the largest clump in the population. The actual value of the projected area occupied by the clumps will be somewhere between these two extremes, and will be determined by the distribution of the clumps inside the cloud. We defer an investigation of how the spatial distribution of discrete over-dense regions affects the reflected X-ray signal to a later study.

\section{Results}

We consider a persistent, unpolarised Sgr A* flare of luminosity $1.3 \times 10^{39} \mathrm{erg} / \mathrm{s}$ (Revnivtsev et al. 2004), modelled with a power law photon index of 1.8 (Baganoff et al. 2001). The photon index assumed in this work allows for a direct comparison with the results of other works on the reflected Sgr B2 X-ray emission (Churazov et al. 2002; Odaka et al. 2011). While more recent works seem to support a somewhat steeper slope $(2.5 \pm 0.3)$ of the incoming spectrum (Porquet et al. 2003, 2008; Nowak et al. 2012), features like the width of the shoulder relative to that of the line, which are important for morphological studies, are not very sensitive to the slope of the initial spectrum. The initial spectrum also wouldn't affect the variation of these features due to variation in the density structures, which are the main focus of this paper. Finally, the inclusion of considerations of the polarisation fraction of the reflected emission can help break some of the degeneracy of the reflected spectrum with the incoming one. We therefore believe the assumption of a 1.8 slope to be justified in this work. We note that, while we assume the source to be unpolarised for simplicity, there is a possibility that X-ray emission of Sgr A* could be partially polarised. In this work, however, we limit the scope of the parameters that we investigated to those of the molecular structure, and postpone the investigation of the effect of the source's initial level of polarisation to a later study.

For each model considered, we plot the energy spectrum and polarisation fraction of the reflected X-ray emission. The polarisation fraction is calculated as the fraction of the Stokes $Q$ parameter (with the frame of reference chosen so that $U=0$ ) intensity over the total intensity reaching the observer.

First, we consider the case where the mass-size relation of the clumps is the Sgr B2 relation discussed in Sect. 3.1.2. We

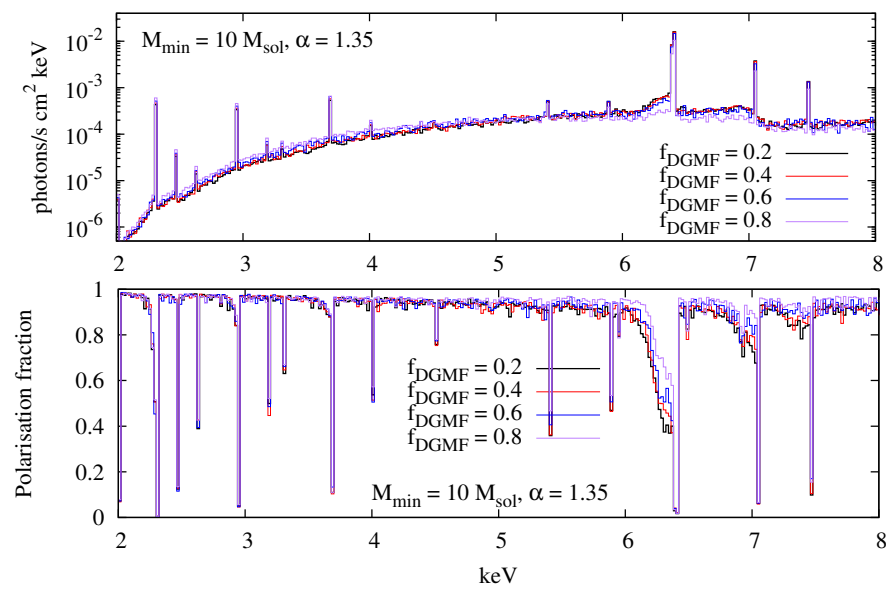

Fig. 4. Reflected energy spectrum and polarisation fraction for varying $f_{\text {DGMF }}$ cloud models. The energy spectrum is shown with a resolution of $20 \mathrm{eV}$.

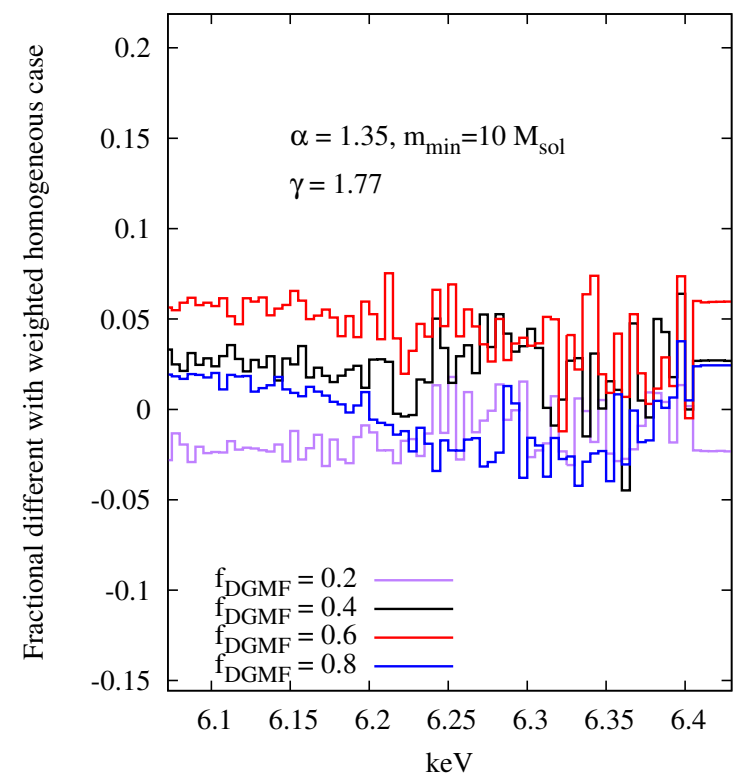

Fig. 5. Fractional difference between the reflected energy spectrum for a cloud model with mass $M_{\mathrm{B} 2}$ and a fraction $f_{\mathrm{DGMF}}$ of its mass found in clumps and a homogeneous cloud model with total mass $M_{\mathrm{B} 2} \times\left(1-f_{\mathrm{DGMF}}\right)$, shown with a spectral resolution of $20 \mathrm{eV}$. The fractional difference is negligible for all energies, reinforcing the idea that the clumping of part of the cloud's mass into very dense regions effectively "hides" that mass from incoming X-rays.

then consider, for fixed $f_{\mathrm{DGMF}}, \alpha$ and $m_{\text {min }}$ parameters, the effect of varying the normalisation of the mass-size relation, and therefore the volume-filling factor of the clump population, on the reflected signal.

\subsection{Fixed Sgr B2 mass-size relation}

We consider a fiducial model given by $f_{\mathrm{DGMF}}=0.4$, $m_{\min }=10 M_{\odot}, \alpha=1.35$, and vary each parameter individually around it while maintaining the mass-size relationship constant at $m_{\text {norm }}=4.68 \times 10^{5} M_{\odot}$ and $\gamma=1.77$ according to Eq. (4).

In Fig. 4, we compare the reflected X-ray emission for different values of the fragmentation parameter $f_{\text {DGMF }}$. For all models considered, we observe that an increase in the fragmentation level of the cloud into clumps results in: a slight increase in 

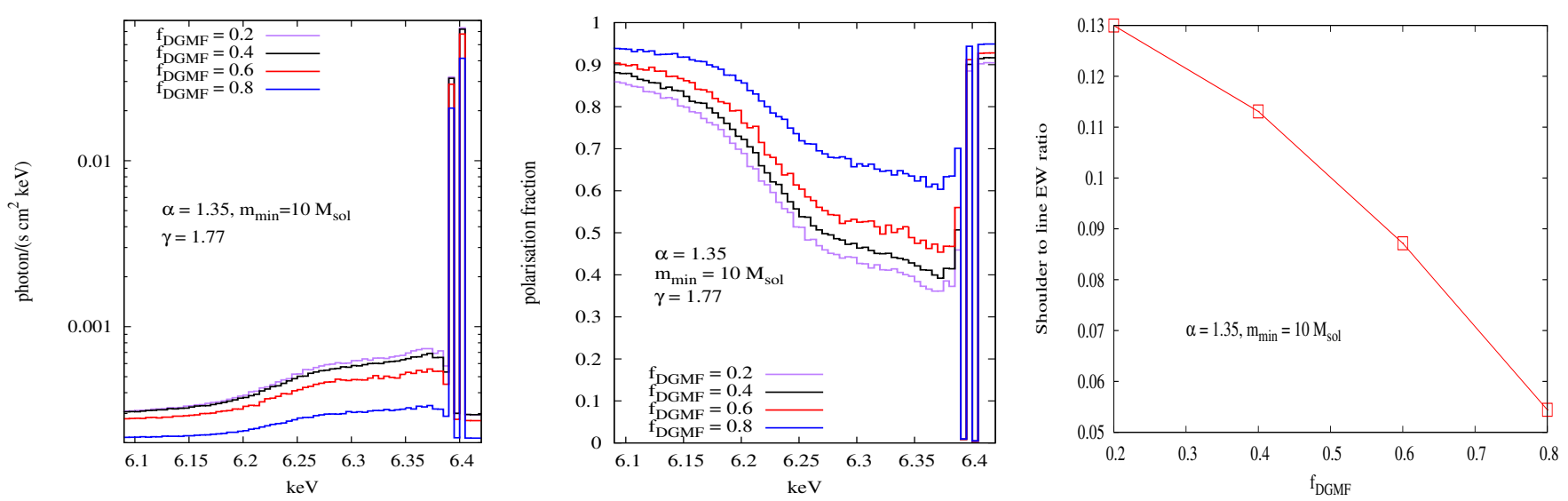

Fig. 6. Reflected energy spectrum and polarisation around the $6.4 \mathrm{keV} \mathrm{Fe} \mathrm{K-} \alpha$ line for varying $f_{\mathrm{DGMF}}$. The spectral resolution is $5 \mathrm{eV}$. The rightmost panel shows the ratio of EW for the $6.4 \mathrm{keV}$ shoulder to the line.

the flux of low energy photons, a decrease in the flux of higher energy photons, and a decrease in the Fe shoulder's flux (see Fig. 6).

These three effects can all be accounted for by considering percolation: because the probability of intercepting the clumps is extremely low, owing to the small $\left(<10^{-4}\right)$ volume-filling fraction of the clumps, the X-ray photons will mainly interact with the atoms and molecules in the interclump medium. The resulting reflected spectra will therefore be consistent with those resulting from reflection off homogeneous clouds with the same size, and density equal to the interclump density. In Fig. 5 we compare the X-ray emission obtained from cloud models where a fraction $f_{\mathrm{DGMF}}$ of the total cloud's mass is found in clumps and homogeneous cloud models with a total mass of $M_{\mathrm{B} 2} \times\left(1-f_{\mathrm{DGMF}}\right)$. We find indeed that the fractional difference between the two cases is negligible for all energies, and that the $f_{\text {DGMF }}=0.2,0.4,0.6$ and 0.8 models can be approximated by homogeneous clouds with $\tau_{\mathrm{HI}} \sim 0.32,0.24,0.16$ and $0.08 \mathrm{re}-$ spectively (where $\tau=R \times n_{\mathrm{HI}} \times \sigma_{\text {Thoms }}$ ).

The decrease in the number of scatterings that resulted from an increase in the dense gas mass fraction can also be observed in the plots of the polarisation fraction of the reflected spectra (Fig. 4). An analytic approximation to the polarisation fraction of an X-ray photon that is undergoing $n$ scatterings is given by (Churazov et al. 2002):

$P_{n}=\frac{1-\eta^{2}}{1+\eta^{2}+\frac{20}{15}\left(\left(\frac{10}{7}\right)^{n-1}-1\right)}$

where $\eta=\cos (\theta)$ and $\theta$ is the average scattering angle. From this analytic prescription, it is indeed for the geometry considered in these calculations, the polarisation fraction should be close to unity for singly scattered photons, in the case of scattering close to $90^{\circ}$ as is the case here, as and should progressively decrease from unity as the number of scatterings increases. With increasing energy the absorption optical depth decreases and the relative contribution to the radiation escaping from the cloud from multiple scatterings increases. This is evident in Fig. 4 as a decrease in the degree of polarisation of the spectrum with increasing energy.

In Fig. 4, we can clearly see that the polarisation fraction of the shoulder progressively increases with the dense gas mass fraction. This means that the higher the fraction of the cloud's gas found in dense regions, the lower the number of multiple scatterings that photons experience, which is in agreement with a picture of an increasing rate of percolation. Fluorescent photons, on the other hand, are emitted isotropically by photoionised atoms, and therefore are completely unpolarised. For varying $\alpha$ and $m_{\text {min }}$ parameters we find that, on the other hand, these have no effect on the overall reflected signal. This reinforces the idea that the dominant effect of clumping within the XRN (in the case of the Sgr B2 mass-size relation) is percolation, and that the mass concentrated in clumps is effectively "hidden" from incoming $\mathrm{X}$-ray photons because of the small volume it occupies.

\subsection{Variable mass-size relation}

Of course the picture painted in Sect. 4.1 of course only holds if the volume-filling fraction of the clump population is low enough to effectively reduce the probability of interaction between photons and overdensities to a negligible value. Should the volume-filling fraction increase, as would result from a variation in the mass-size relation of the clumps (see Sect. 3.1.2), then $\mathrm{X}$-rays should start intercepting the clumps at a more significant rate, with consequences to the reflected energy and polarisation spectrum.

In particular, an increase in the absorption probability should result in an increase in the fluorescent lines, while an increase in the scattering probability should result in an increase in the fraction of fluorescent photons scattered, and therefore of the flux of the fluorescent line's shoulder. In Fig. 7, we show the energy and polarisation spectrum around the $6.4 \mathrm{keV} \mathrm{K}-\alpha$ line for cases of increasing high volume-filling factor. We find that, as expected, both the line and the shoulder's flux increase with increasing $\mu$.

Once the probability of intercepting clumps increases, we expect properties of the clump populations such as $m_{\min }$ and $\alpha$ to play a more significant role in shaping the reflected X-ray signal. In Fig. 8, we compare the reflected signal in the case of varying $\alpha$ and $m_{\min }$ parameters, respectively, for fixed $\mu=0.01$. Indeed, for this volume-filling fraction we already observe that the slope shows a dependence on the two population parameters: a higher CMF slope results in a higher number of clumps with larger masses (and hence radii) being selected. Because these are more likely to intercept the incident X-rays, we expect an increase in the Fe shoulder's flux in correspondence with increasing $\alpha$, as it is indeed observed in the figure. A decrease in $m_{\min }$ puts more mass in smaller clumps, resulting in a decrease in the fluorescent lines and shoulders in Fig. 8. However, a photon which is emitted inside a denser clump is also more likely 
M. Molaro et al.: Internal structure of XRNe
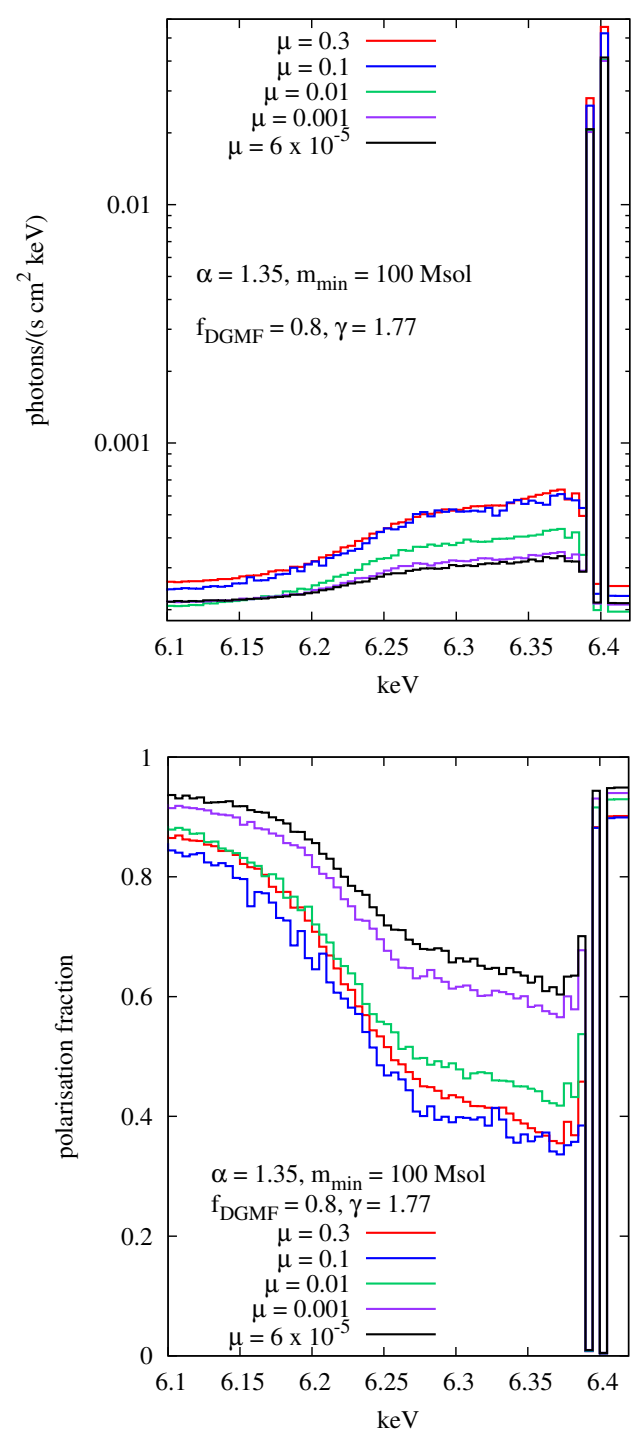

Fig. 7. Reflected energy (top plot) and polarisation (bottom plot) around the $6.4 \mathrm{keV} \mathrm{Fe} \mathrm{K-} \alpha$ line for varying $\mu$, shown with a resolution of $5 \mathrm{eV}$. The values of $\mu$ illustrated were obtained by adjusting the $m_{\text {norm }}$ parameter in the mass-relation of clumps using Eq. (5). As expected, for increasingly large volume-filling factors, the probability of fluorescent photons intercepting the clumps increases, resulting in an increase in the shoulder's flux. Note that, because the X-ray signal is really dependent on the projected area (see Sect. 3.1.4), which roughly goes as $\mu^{2 / 3}$, we would expect a volume-filling factor of $\mu \sim 0.001$ (project area $\sim 0.01$ ) to already produce a visible signature in the X-ray spectrum. Indeed, from the polarisation fraction plot, it is clear that at $\mu=0.001$ the signal starts deviating from the virtually homogeneous case.

to be scattered before it escapes, and therefore the ratio of the shoulder to line increases with decreasing $m_{\min }$, as seen in the equivalent width $(\mathrm{EW})$ ratio plot in the same figure.

\section{Time-evolution of the XRN morphology as a probe of the 3D distribution of substructures}

As a result of the finite speed of light, the illumination by a flare of duration shorter than the light-crossing time of the cloud results in different regions of the GMC being visible to the observer, in the form of reflected X-ray emission, at different times. The evolution of the reflected X-ray intensity, therefore, acts as a scan of the density structure of the cloud as the wavefront propagates through it (Sunyaev \& Churazov 1998). In this section we discuss the importance of this effect in the context of the study of the GMC's clump properties and distribution.

For these calculations we focus on three of the Sgr B2 models that were considered in the previous sections:

- the fiducial model, which assumes parameters $f_{\text {DGMF }}=0.4$, $m_{\min }=10 M_{\odot}, \alpha=1.35$ and the Sgr B2 mass-size relation that is consistent with observations of real Sgr B2 clumps (see Sect. 3.1.2), which corresponds to a volume-filling fraction of $\mu \sim 1.3 \times 10^{-5}$. We refer to this model as the Sgr B2 mass-size model;

- the homogeneous model, where we assume no clumps at all;

- a more "visible" clump population model, which considers a case in which most of the gas $\left(f_{\mathrm{DGMF}}=0.8\right)$ is contained in relatively massive $\left(m_{\min }=100 M_{\odot}\right)$ clumps, which are described by a mass-size relation that is constrained to obtain a volume-filling fraction as large as $\mu=0.01$ (see Sect. 3.1.2). This case ensures clumps will be numerous and voluminous enough to be easily recognisable in our calculations. We refer to this model as the " $\mu=0.01$ " model.

While these calculations were being performed, NuSTAR was able to resolve the Sgr B2 clumps Sgr B2(N) and Sgr B2(M) in X-rays for the first time (Zhang et al. 2015). This new result shows the feasibility and potential that high-resolution studies of the X-ray morphology of GMCs in the CMZ have in the study of the internal structure of these XRNe. We emphasise, however, that the mass-size relation assumed in our Sgr B2 masssize model makes use of the Qin et al. (2011) observations of the clumps, which were able to resolve the Sgr B2(N) and Sgr B2(M) clumps into distinct and independent substructures. The clumps for this model that we obtained in our simulation are therefore more compact than the region of gas considered by the Zhang et al. (2015) observations.

In a single scattering approximation, the distance $D$ along the line of sight $(l, b)$ at which light has to be scattered to reach the observer at time $t$, defined such that $t=0$ is the time at which the flare was last observed directly, is given by

$D\left(t, t^{\prime}\right)=\frac{c t^{2}-O_{x}^{2}+2 c t\left(\left|O_{x}\right|-t^{\prime} c\right)+\left(|O x|-t^{\prime} c\right)^{2}}{2\left(c t+\left(\left|O_{x}\right|-t^{\prime} c\right)+O_{x} \cos (b) \cos (l)\right)}$

where $O_{x}=-8 \mathrm{kpc}$ is the Sun's location with respect to the emitting source (assuming $O_{y}=O_{z}=0$ ), and $-T \leq t^{\prime} \leq 0$ is the time during the flare of duration $T$ at which the photon was emitted, as illustrated in Fig. 9. The region illuminated at a given time is therefore an ellipsoid, with its focus at the observer's position. For the case of an observer located at the Sun's position, this can be approximated, in the proximity of Sgr B2, by a paraboloid (Cramphorn \& Sunyaev 2002). The propagation of the section of the ellipsoid on the $x-y$ plane is illustrated in 10 for the case of an instantaneous flare $(T=0)$.

In the case of a flare with finite duration, i.e. $T>0$, the duration of the flare determines the "thickness" of the ellipsoid, or in other words the thickness of the region that is simultaneously visible to the observer, as illustrated in Fig. 10. The surface brightness observed along a line of sight at a given moment, $I(l, b, t)$, will therefore be determined not by the total optical depth of the cloud in that direction, but rather by the surface density in the section of the cloud that is delimited by the thick paraboloid (Sunyaev \& Churazov 1998), whose boundaries are determined by the beginning and end of the flare. The reflected 

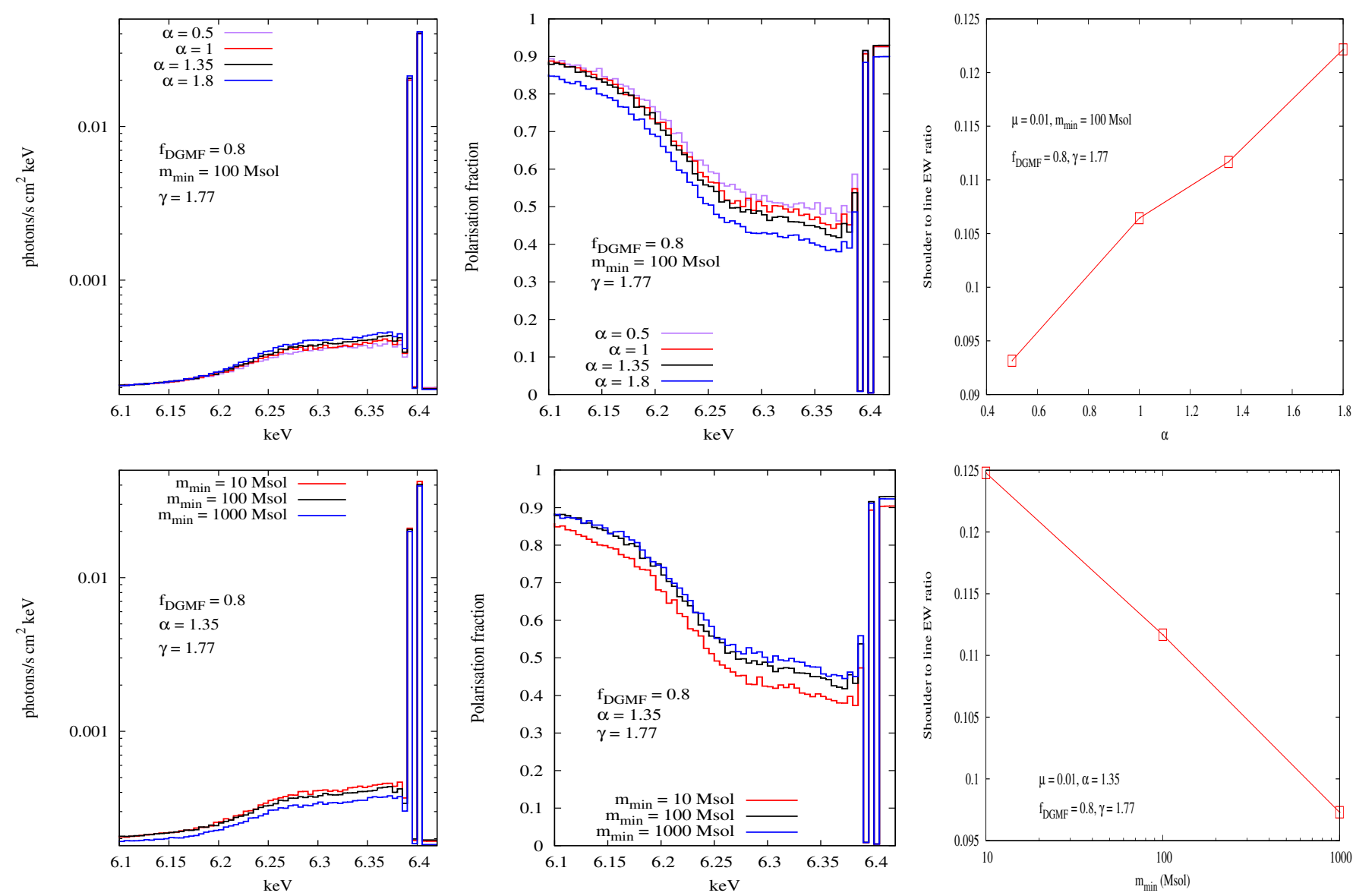

Fig. 8. Reflected energy (left plots) and polarisation (centre plots) around the $6.4 \mathrm{keV} \mathrm{Fe} \mathrm{K-} \alpha$ line (shown with resolution of $5 \mathrm{eV}$ ) and shoulder to line ratio (right plots) for varying $\alpha$ (top plots) and $m_{\min }$ (bottom plots) parameters at fixed $\mu=0.01$. Values of $\mu$ illustrated were obtained by adjusting the $m_{\text {norm }}$ parameter in the mass-relation of clumps using Eq. (5). For increasing $\alpha$, we expect an increase in the number of clumps being sampled from the higher mass range, resulting in a greater average size of the clumps in the population. In return, this results in an increase in the probability of X-rays intercepting them. Indeed, an increase in the Fe shoulder for increasing $\alpha$ is observed both in the energy and polarisation spectrum. The fragmentation of the clumps to lower and lower $m_{\min }$, on the other hand, results in a larger projected area of the clump population, which increases the probability of interaction with incoming X-rays. This effect in also observed in the plots.

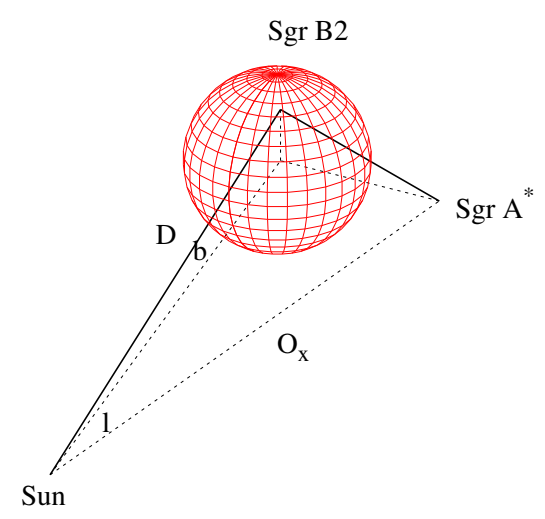

Fig. 9. Schematic representation (not to scale) of the parameters used in Eq. (9).

intensity can therefore be described, under a single scattering approximation, as:

$I(l, b, t, v)=\int_{-T}^{0} \sum_{Z} \frac{\rho(v)}{4 \pi R^{2}} n_{Z} \frac{\mathrm{d} \sigma_{Z}}{\mathrm{~d} \Omega} \exp \left(-\tau_{Z}\right) c \mathrm{~d} t^{\prime}$

where $\rho(v)$ is the number of photons/(s keV) emitted by the source, $R$ is the distance from the source to the point of scattering, $n_{z}$ is the density at the point of scattering, $\frac{\mathrm{d} \sigma_{Z}}{\mathrm{~d} \Omega}$ is the singly-differentiated cross-section, computed using the public library xraylib (Schoonjans et al. 2011) and:

$\tau_{Z}=\operatorname{NHI}\left(\sigma_{\mathrm{abs}, Z}+\sigma_{\text {scatt }, Z}\right)$

is the total optical depth, from the point of emission to the point of observation. To speed-up the calculations, we ignore the contribution of Raman scattering to $\sigma_{\text {scatt }}$, whose contribution is negligible for this particular geometrical set-up (i.e. for an average scattering angle of $\sim 90^{\circ}$ ).

Owing to their higher average density, clumps are able to contribute significantly towards $I$, by scattering more X-ray flux towards the observer compared to the interclump medium, and hence should be clearly recognisable in the morphology of the XRN at times when they are intercepted by the propagating paraboloid, as first suggested by Sunyaev \& Churazov (1998). Once the paraboloid has passed them, the clumps should significantly contribute towards the intervening column density NHI, and therefore still be visible in the morphology of the XRN as regions of absorption.

In Fig. 11, we illustrate this in the case of the three clump models that are described at the beginning of the section, both in the case of a short $(T=1 \mathrm{yr})$ and longer $(T=20 \mathrm{yr})$ flare, in a snapshot at time $t=320 \mathrm{yr}$. The intensity and column density 

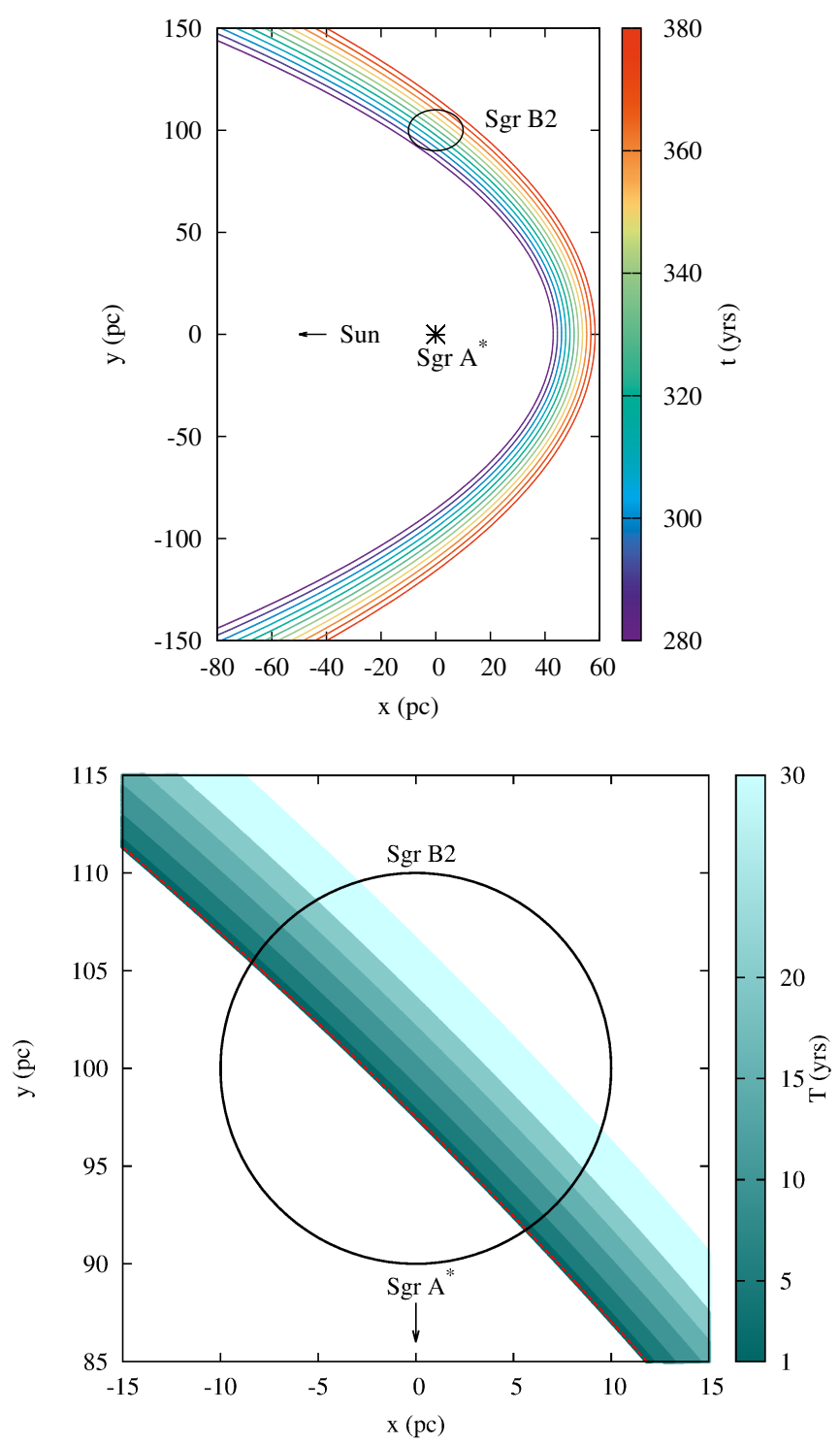

Fig. 10. Top plot: regions of the $x-y$ plane visible to the observer at different times through scattered X-ray photons, in the case of photons originally emitted by an instantaneous flare of Sgr A* (i.e. all photons emitted at $t=0$ ). Bottom plot: regions of the $x-y$ plane visible to the observer through scattered X-rays at time $t=320 \mathrm{yr}$, in the case of an instantaneous flare (dashed line) and in the case of flares of duration $T$ (coloured maps). The longer the duration of the flare, the thicker the region of the sky observable simultaneously.

for the equatorial, vertical and edge profiles that are indicated on the maps are shown in Figs. 12 and 13, respectively.

On the maps, the main visible effects are as follows:

- the contribution of clumps towards the scattered intensity is indeed clearly visible in the form of bright spots, consistently with the findings of Zhang et al. (2015);

- intervening clumps located between the source and the point of scattering, or between the point of scattering and the observer (see Fig. 13) contribute considerably to the absorption of the X-ray radiation, and are, therefore, observable as regions of absorption in the maps;

- the effect of the duration of the flare is also recognisable: the longer the duration of the flare, the thicker the region of the cloud that is probed by the ellipsoid at the same time, hence the higher the number of clumps that are probed by the paraboloid simultaneously, as clearly seen in the $\mu=$ 0.01 model maps;

- in the case of clumps with Sgr B2 mass-size relation, the very small volume they occupy means the probability of a short flare intercepting them is very low, and in fact very few clumps are intercepted at all for this particular distribution, and most clumps are therefore only seen in absorption;

- the intensity of the interclump regions in the Sgr B2 masssize model (average density $n_{\mathrm{H} 2} \sim 1 \times 10^{3} \mathrm{~cm}^{-3}$ ), is higher than that of the homogeneous model (average density $n_{\mathrm{H} 2} \sim$ $1 \times 10^{4} \mathrm{~cm}^{-3}$ ) in the central region of the cloud. This is due to the fact that, although contributing a larger surface density within the thick paraboloid, the homogeneous cloud also results in a larger absorbing column density, as shown in Fig. 13.

The reflected intensity at a given time can therefore reveal information on the column density of both the clump and the interclump medium. But it also contains information on the distribution of the clumps inside the cloud.

In the case of a short flare, the distance along the line of sight that is observed at a given time is uniquely defined by the time of observation, since $D\left(t, t^{\prime}\right) \sim D\left(t, t^{\prime}=0\right)$. In this case, by comparing the time at which a clump becomes visible on the reflected intensity map with its location on the sky, it is possible to constrain its position $D$ along the line of sight, as illustrated in Fig. 14. This kind of analysis could therefore prove to be important when studying the 3D distribution of substructures within GMCs.

For flares of finite duration, on the other hand, a range of distances will be observable at the same time along a given line of sight, as photons are emitted by the source over a period of time $T$, which determines the "thickness" of the region observed at a given time. In the case of the known duration of flares, as is the case for many X-ray sources in the Galaxy, the timeevolution of the reflected intensity could still be used in this kind of analysis. On the other hand, in the case of illumination by a flare of unknown duration, on the other hand, it would be impossible to constrain the distance $D$ of each clump along its line of sight, since the range of possible values will be proportional to the duration of the flare itself. On the other hand, if the position of at least two clumps is known, it would be possible to reverse the problem and infer a lower-bound to the duration of the flare, as illustrated in Fig. 15.

The visibility of clumps in the reflected X-ray intensity, their localised nature, and the non-persistent illumination from external flaring sources such as Sgr A*, make the time-evolution of the X-ray morphology of Sgr B2 and similar XRNe an ideal target in the study of the spatial distribution of clumps within them. We leave the study of the intensity light curve of individual clumps, as a function of photon energy, for different clump sizes and optical depths, for future work.

\section{Conclusions}

We studied the effect of clumps on the X-ray emission of GMCs that act as XRNe by modelling Sgr B2, one of the brightest and most massive XRNe in our Galaxy.

In particular, we studied the effect of the internal structure of GMCs on the properties of X-ray spectrum, polarisation and morphology reflected from them. We have considered both persistent sources and transients, in particular giant flares, as the 
A\&A 589, A88 (2016)
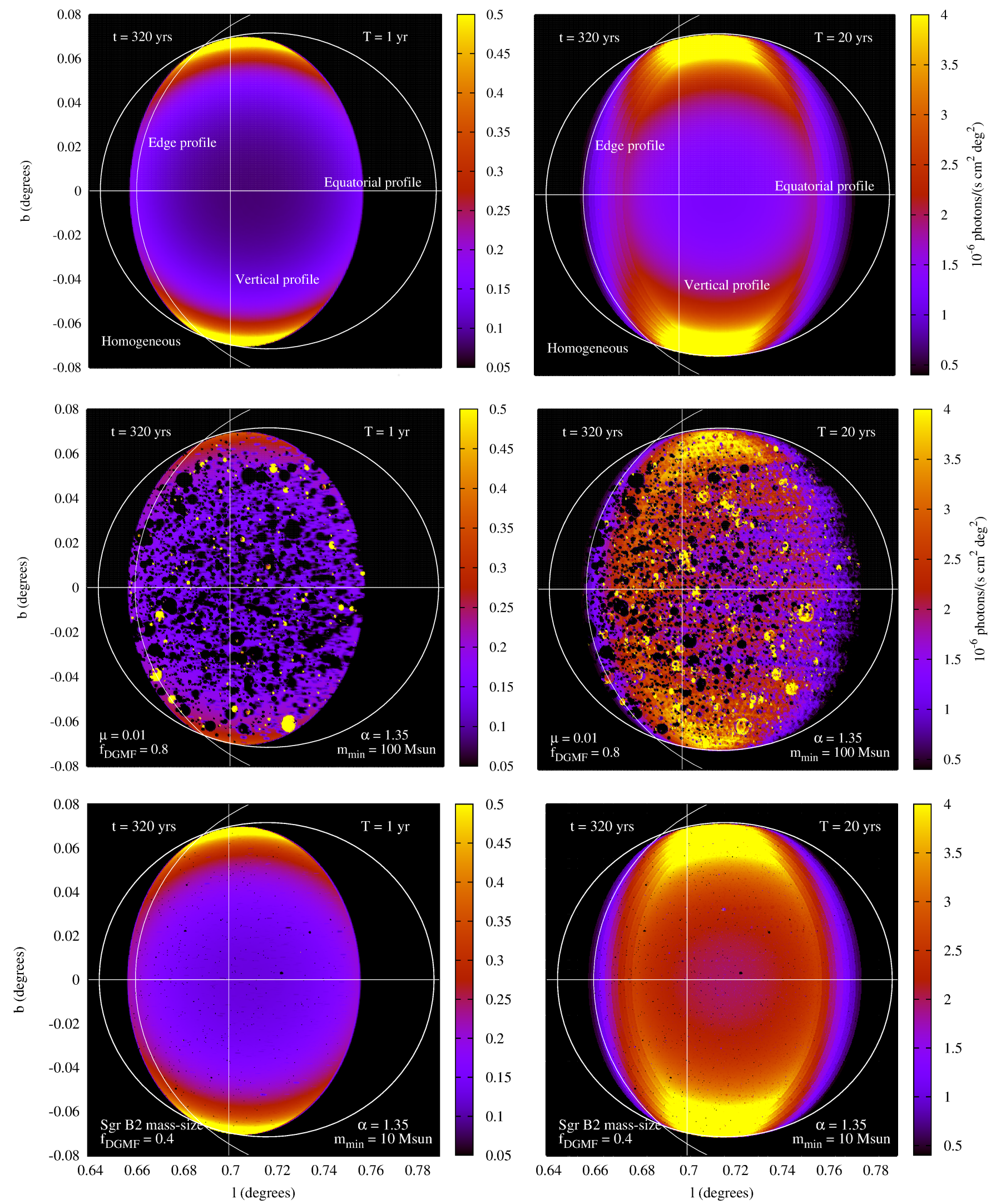

Fig. 11. Analytic, single scattering approximation of the $3-20 \mathrm{keV}$ reflected $\mathrm{X}$-ray intensity observed at time $t=320 \mathrm{yr}$ for the three cloud models, discussed in the text, and for the case of a short $(1 \mathrm{yr})$ and long $(20 \mathrm{yr})$ flare. The source, located at $l=0^{\circ}, b=0^{\circ}$, illuminates the cloud from the left side in the above plots. The angular resolution used is of 1 arcsec. The intensity and column density profiles for the equatorial, vertical, and edge profiles indicated in the maps are shown in Figs. 12 and 13, respectively. 
M. Molaro et al.: Internal structure of XRNe
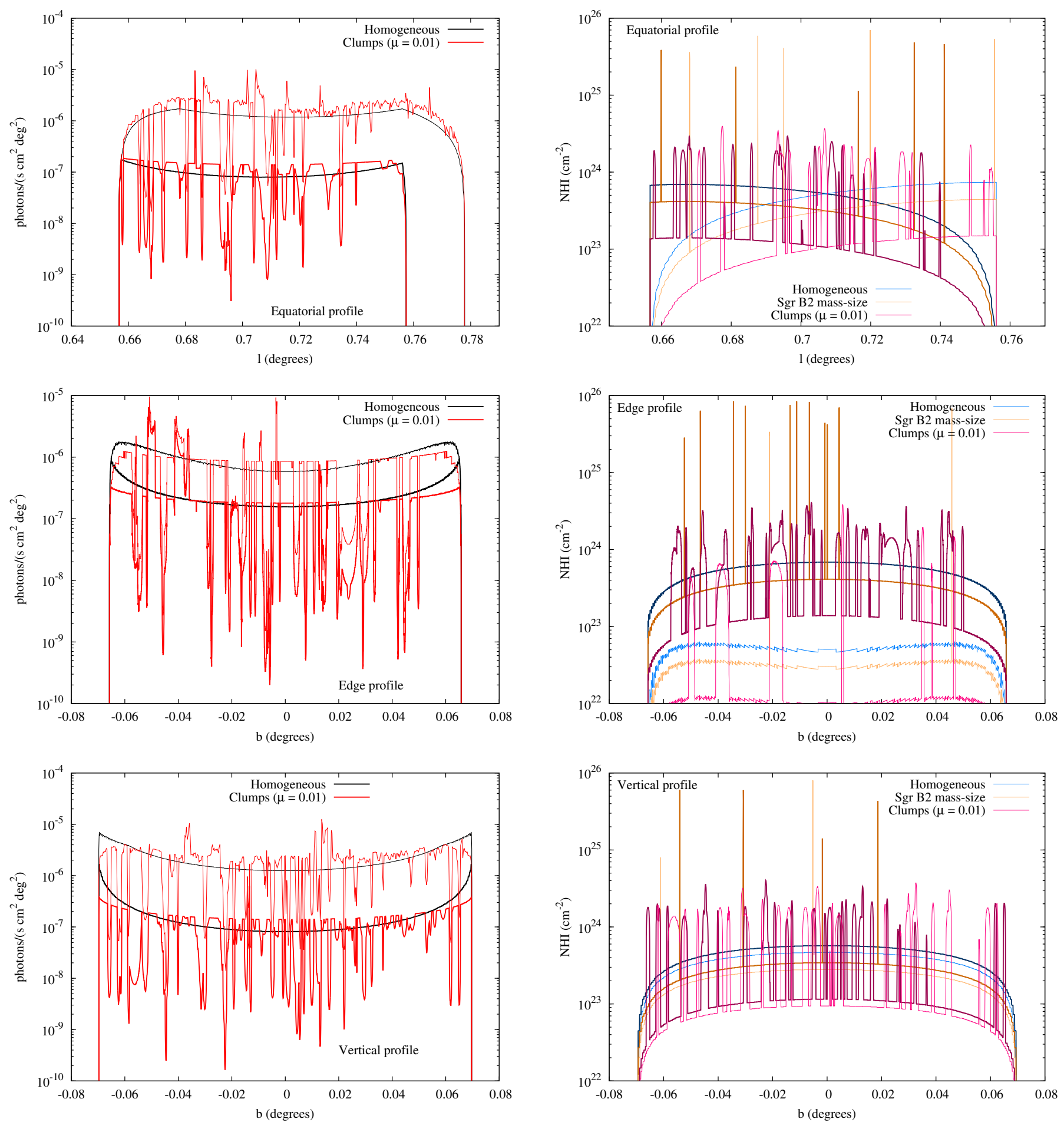

Fig. 12. Scattered intensity profiles for the homogeneous and $\mu=$ 0.01 model for the $T=1 \mathrm{yr}$ (thick lines) and $T=20 \mathrm{yr}$ (thin lines).

source of incident X-rays. We use Sgr B2 as a case study, but most of our results are generally applicable to any GMC in the Galaxy. We defined a simple clump model for simplicity. We investigated the effect of different clump population model parameters on the reflected X-ray energy and polarisation spectrum. The parameters that we investigated included the fraction of the total mass of the cloud contained in clumps $\left(f_{\mathrm{DGMF}}\right)$, the slope of the clump mass function $(\alpha)$, the minimum mass of clumps found in the population $\left(m_{\min }\right)$, and the mass-size relation of individual clumps $\left(m=m_{\text {norm }}(r / p c)^{\gamma}\right)$. First, we considered a

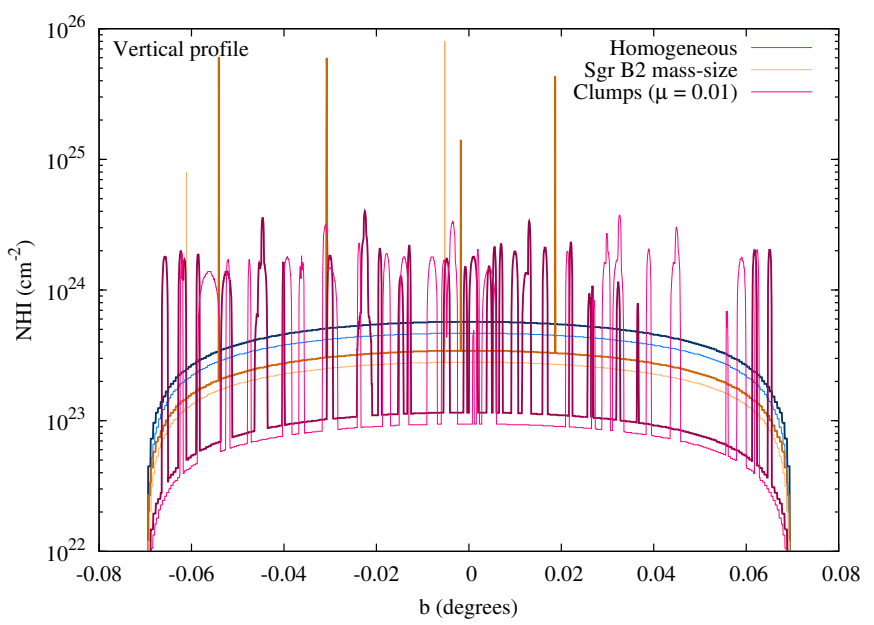

Fig. 13. Column density from the source to the point of scattering (darker colours) and from the point of scattering to the observer (lighter colours) along profiles indicated in Fig. 11, excluding the contribution of the diffuse envelope. The plots show the case of minimum column density $\left(t^{\prime}=0\right)$ at time $t=320$ yrs. The different mass-size relations assumed in clump models "Sgr B2 mass-size" and " $\mu=0.01$ " result in the latter case presenting: a higher probability of intercepting clumps, broader absorption features in the intensity profiles, and lower column density peaks.

fixed mass-size relation that was consistent with the clumps observed in Sgr B2, and varied each of the remaining parameters around a fiducial model given by ${ }_{\mathrm{DGMF}}=0.4, m_{\min }=10 \mathrm{M}_{\odot}$, 

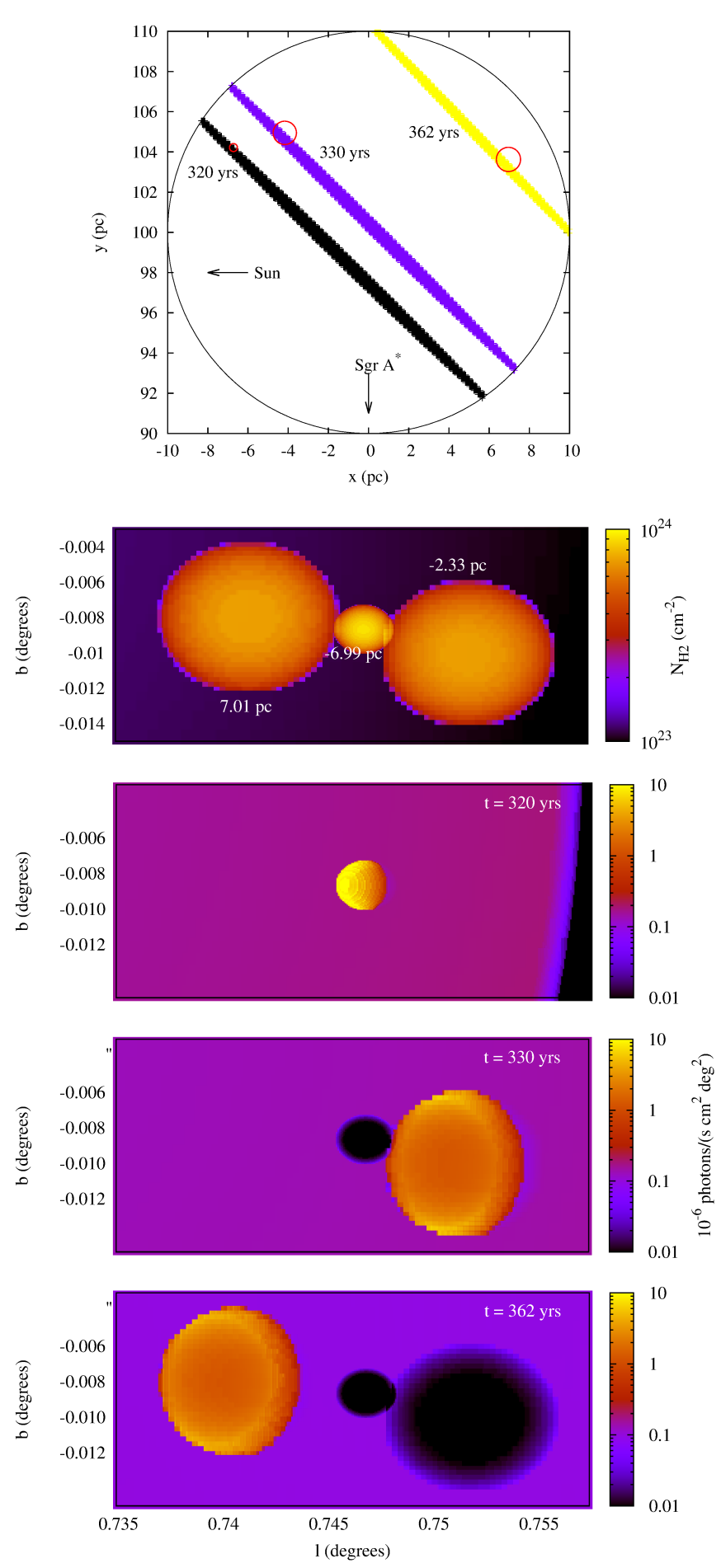

Fig. 14. Top plot: projected location of the clumps inside Sgr B2 and regions inside Sgr B2 visible to the observer at times when the substructures should become visible (assuming illumination by an instantaneous flare). Second plot: column density map of three simulated clumps within Sgr B2, found in a small region of the sky (labels in plot indicate the clumps' distance from the centre of the cloud, approximated as $\left.D-D_{\mathrm{SgrB} 2}\right)$. Third, fourth and fifth plots: reflected X-ray intensity reaching the observer at different times. Despite the apparent proximity of the clumps on the sky, because of the different distance at which they are located along the line of sight, the clumps are visible through their reflected X-ray emission at different times, so that when one is visible, the others are not.

and $\alpha=1.35$, assessing their effect on the overall reflected X-ray spectrum.

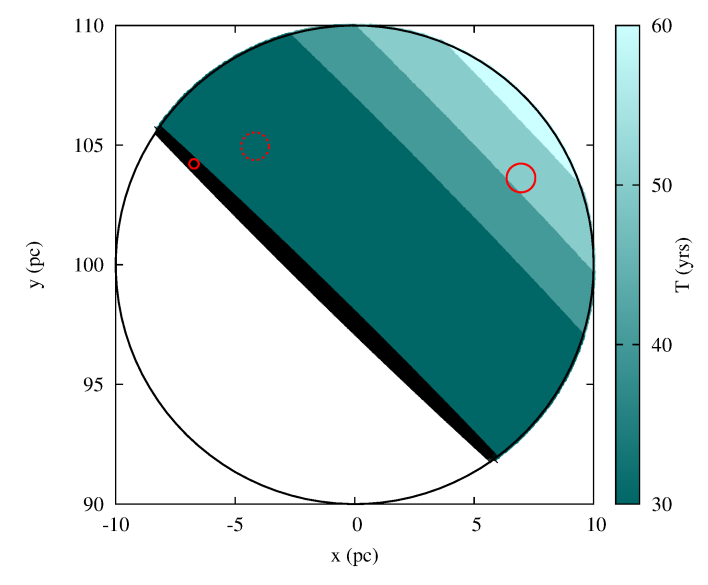

Fig. 15. Projected regions inside the cloud observed simultaneously at time $320 \mathrm{yr}$ by flares of different durations $T$ (where the black region corresponds to illumination by an instantaneous flare). In the case of the three clumps considered in Fig. 14, the minimum flare duration required for both the furthest apart clumps (shown with solid red lines in the plot) to be visible to the observer at the same time is $T \sim 40 \mathrm{yr}$. In the case in which both substructures are visible simultaneously in the reflected X-ray intensity maps, one can infer that the source's flare must have lasted at least $T \sim 40 \mathrm{yr}$.

In this case, the volume-filling fraction of the clumps, and therefore the relative probability of X-rays being scattered by gas in clumps compared to the interclump medium, is negligible. The cloud therefore appears in X-rays as having a mass smaller than the total mass by the amount that is clumped. The extremely low volume-filling fraction, which was obtained when assuming the mass-size relation observed in Sgr B2, allows these clumps to effectively "hide" a fraction $f_{\text {DGMF }}$ of the cloud's mass in an extremely small fraction of the cloud's volume. We explicitly check this hypothesis by considering the case of homogeneous clouds containing $\left(1-f_{\text {DGMF }}\right)$ of the cloud's original mass and no clumps at all. In cases where the mass-size relation of clumps means these occupy a much higher volume-filling fraction, we find that clumps do contribute towards reflection, and that the reflected X-rays contain information about the internal structure of the cloud. The parameters of the clumping model could therefore be constrained by X-ray observations.

We also investigated how the time evolution of the spatiallyresolved images of the reflected X-ray intensity can be used to probe the location of individual substructures along the line of sight in the case where the incident X-rays have a transient origin, such as a short-duration flare from a X-ray binary or the supermassive black hole at the centre of our Galaxy. We have shown that in the case of transient sources, the timing information, retrievable both in emission and in absorption, can be used to probe the third dimension along the line of sight, opening up the possibility of 3D tomography of the cloud. Future X-ray observatories such as Astro-H (Takahashi et al. 2010), Athena (Barcons et al. 2012), and the X-ray Surveyor (Weisskopf et al. 2015) could therefore open up a new probe of the internal structure of GMCs. The recent pre-selection of the X-ray polarimeters IXPE (Weisskopf et al. 2014), XIPE (Soffitta et al. 2013), PRAXyS (Jahoda et al. 2015) or the XTP (Wang et al. 2014) instruments could further complement spectrographic studies to polarimetric ones.

Acknowledgements. We thank the referee Frédéric Marin for his helpful comments on the draft. We also thank Eugene Churazov and Diederik Kruijssen for discussions. 


\section{References}

Allemand, C. D. 1970, App. Spectrosc., 24, 348

Baganoff, F. K., Bautz, M. W., Brandt, W. N., et al. 2001, Nature, 413, 45

Barcons, X., Barret, D., Decourchelle, A., et al. 2012, ArXiv e-prints [arXiv: 1207.2745]

Battisti, A. J., \& Heyer, M. H. 2014, ApJ, 780, 173

Capelli, R., Warwick, R. S., Porquet, D., Gillessen, S., \& Predehl, P. 2012, A\&A, 545, A35

Chen, C.-Y., \& Ostriker, E. C. 2015, ApJ, 810, 126

Churazov, E., Sunyaev, R., \& Sazonov, S. 2002, MNRAS, 330, 817

Clavel, M., Terrier, R., Goldwurm, A., et al. 2013, A\&A, 558, A32

Cramphorn, C. K., \& Sunyaev, R. A. 2002, A\&A, 389, 252

Dogiel, V., Cheng, K.-S., Chernyshov, D., et al. 2009, PASJ, 61, 901

Donkov, S., Veltchev, T. V., \& Klessen, R. S. 2011, MNRAS, 418, 916

Draine, B. T. 2011, Physics of the Interstellar and Intergalactic Medium ed. B. T. Draine (Princeton University Press)

Etxaluze, M., Goicoechea, J. R., Cernicharo, J., et al. 2013, A\&A, 556, A137

Gando Ryu, S., Nobukawa, M., Nakashima, S., et al. 2013, PASJ, 65, 33

Ginsburg, A., Bally, J., Battersby, C., et al. 2015, A\&A, 573, A106

Gordon, M. A., Berkermann, U., Mezger, P. G., et al. 1993, A\&A, 280, 208

Haardt, F., \& Matt, G. 1993, MNRAS, 261, 346

Hasegawa, T., Sato, F., Whiteoak, J. B., \& Miyawaki, R. 1994, ApJ, 429, L77

Inui, T., Koyama, K., Matsumoto, H., \& Tsuru, T. G. 2009, PASJ, 61, 241

Jahoda, K., Kouveliotou, C., Kallman, T. R., \& Praxys Team 2015, in AAS Meeting Abstracts, 225, 338.40

Kauffmann, J., Pillai, T., Shetty, R., Myers, P. C., \& Goodman, A. A. 2010, ApJ, 716,433

Könyves, V., André, P., Men'shchikov, A., et al. 2010, A\&A, 518, L106

Kruijssen, J. M. D., Longmore, S. N., Elmegreen, B. G., et al. 2014, MNRAS, 440, 3370

Langer, W. D., Goldsmith, P. F., Pineda, J. L., et al. 2015, A\&A, 576, A1

Larson, R. B. 1981, MNRAS, 194, 809

Lis, D. C., \& Goldsmith, P. F. 1990, ApJ, 356, 195

Lodders, K. 2003, ApJ, 591, 1220

Lombardi, M., Alves, J., \& Lada, C. J. 2010, A\&A, 519, L7

Marin, F. 2015, ArXiv e-prints [arXiv: 1508.00405]

Marin, F., Karas, V., Kunneriath, D., \& Muleri, F. 2014a, MNRAS, 441, 3170

Marin, F., Karas, V., Kunneriath, D., Muleri, F., \& Soffitta, P. 2014b, in SF2A2014, Proc. of the Annual Meeting of the French Society of Astronomy and Astrophysics, eds. J. Ballet, F. Martins, F. Bournaud, R. Monier, \& C. Reylé, 109

Marin, F., Muleri, F., Soffitta, P., Karas, V., \& Kunneriath, D. 2015, A\&A, 576, A19

McKee, C. F., \& Ostriker, E. C. 2007, ARA\&A, 45, 565

Meagher, D. 1980, Technical Report IPL-TR-80-11 I

Meszaros, P., Novick, R., Szentgyorgyi, A., Chanan, G. A., \& Weisskopf, M. C. 1988, ApJ, 324, 1056

Motte, F., Andre, P., \& Neri, R. 1998, A\&A, 336, 150

Muno, M. P., Baganoff, F. K., Brandt, W. N., Park, S., \& Morris, M. R. 2007, ApJ, 656, L69

Murakami, H., Koyama, K., Sakano, M., Tsujimoto, M., \& Maeda, Y. 2000, ApJ, 534,283

Murakami, H., Koyama, K., Tsujimoto, M., Maeda, Y., \& Sakano, M. 2001, ApJ, 550,297

Namito, Y., Ban, S., \& Hirayama, H. 1993, Nucl. Instruments Meth. Phys. Res. A, 332, 277
Nobukawa, M., Ryu, S. G., Tsuru, T. G., \& Koyama, K. 2011, ApJ, 739, L52

Nowak, M. A., Neilsen, J., Markoff, S. B., et al. 2012, ApJ, 759, 95

Nutter, D., \& Ward-Thompson, D. 2007, MNRAS, 374, 1413

Odaka, H., Aharonian, F., Watanabe, S., et al. 2011, ApJ, 740, 103

Parsons, H., Thompson, M. A., Clark, J. S., \& Chrysostomou, A. 2012, MNRAS 424, 1658

Ponti, G., Terrier, R., Goldwurm, A., Belanger, G., \& Trap, G. 2010, ApJ, 714, 732

Ponti, G., Morris, M. R., Terrier, R., \& Goldwurm, A. 2013, in Cosmic Rays in Star-Forming Environments, eds. D. F. Torres, \& O. Reimer, Advances in Solid State Physics, 34, 331

Ponti, G., Morris, M. R., Clavel, M., et al. 2014, in IAU Symp. 303, eds. L. O. Sjouwerman, C. C. Lang, \& J. Ott, 333

Porquet, D., Predehl, P., Aschenbach, B., et al. 2003, A\&A, 407, L17

Porquet, D., Grosso, N., Predehl, P., et al. 2008, A\&A, 488, 549

Poutanen, J., \& Vilhu, O. 1993, A\&A, 275, 337

Pozdnyakov, L. A., Sobol, I. M., \& Syunyaev, R. A. 1983, Astrophys. Space Phys. Rev., 2, 189

Qin, S.-L., Schilke, P., Rolffs, R., et al. 2011, A\&A, 530, L9

Revnivtsev, M. G., Churazov, E. M., Sazonov, S. Y., et al. 2004, A\&A, 425, L49

Salpeter, E. E. 1955, ApJ, 121, 161

Schoonjans, T., Brunetti, A., Golosio, B., et al. 2011, Spectrochim. Acta B, 66, 776

Shetty, R., Collins, D. C., Kauffmann, J., et al. 2010, ApJ, 712, 1049

Soffitta, P., Barcons, X., Bellazzini, R., et al. 2013, Exper. Astron., 36, 523

Sunyaev, R., \& Churazov, E. 1998, MNRAS, 297, 1279

Sunyaev, R. A., \& Churazov, E. M. 1996, Astron. Lett., 22, 648

Sunyaev, R. A., Markevitch, M., \& Pavlinsky, M. 1993, ApJ, 407, 606

Sunyaev, R., Gilfanov, M., \& Churazov, E. 1999, in Highlights in X-ray Astronomy, eds. B. Aschenbach, \& M. J. Freyberg, 272, 102

Szymanski, H. 1970, Raman Spectroscopy: Theory and Practice, 1st edn. (Donington House, 30 Norfolk Street, London, WC2, England: Plenum Press), 2

Takahashi, T., Mitsuda, K., Kelley, R., et al. 2010, in SPIE Conf. Ser., 7732

Taverna, R., Muleri, F., Turolla, R., et al. 2014, MNRAS, 438, 1686

Terrier, R., Ponti, G., Bélanger, G., et al. 2010, ApJ, 719, 143

Tsuboi, M., \& Miyazaki, A. 2012, PASJ, 64, 111

Vainshtein, L. A., Syunyaev, R. A., \& Churazov, E. M. 1998, Astron. Lett., 24, 271

Valinia, A., Tatischeff, V., Arnaud, K., Ebisawa, K., \& Ramaty, R. 2000, ApJ, 543,733

Verner, D. A., \& Yakovlev, D. G. 1995, A\&AS, 109, 125

Wang, Z., Shen, Z., Mu, B., et al. 2014, in SPIE Conf. Ser., 9144, 1

Weisskopf, M. C., Bellazzini, R., Costa, E., et al. 2014, in AAS/High Energy Astrophysics Division, 14, 116.15

Weisskopf, M. C., Gaskin, J., Tananbaum, H., \& Vikhlinin, A. 2015, in SPIE Conf. Ser., 9510, 2

Williams, J. 1999, in Interstellar Turbulence, eds. J. Franco, \& A. Carraminana, 190

Williams, J. P., Blitz, L., \& McKee, C. F. 2000, Protostars and Planets IV, 97

Yusef-Zadeh, F. 2013, in Cosmic Rays in Star-Forming Environments, eds. D. F. Torres, \& O. Reimer, Astrophys. Space Sci. Proc., 34, 325

Yusef-Zadeh, F., Law, C., \& Wardle, M. 2002, ApJ, 568, L121

Zhang, S., Hailey, C. J., Mori, K., et al. 2015, ApJ, 815, 132 